\title{
On Quadruple Random Fixed Point Theorems in Partially Ordered Metric Spaces
}

\author{
R. A. Rashwan and D. M. Al-Baqeri \\ Department of Mathematics, University of Assiut, P.O. Box 71516, Assiut, Egypt \\ Correspondence should be addressed to R. A. Rashwan; rr_rashwan54@yahoo.com
}

Received 18 July 2013; Accepted 8 October 2013; Published 30 January 2014

Academic Editors: F. Bobillo, M. Coppens, Z. Gong, and S. Zhang

Copyright (C) 2014 R. A. Rashwan and D. M. Al-Baqeri. This is an open access article distributed under the Creative Commons Attribution License, which permits unrestricted use, distribution, and reproduction in any medium, provided the original work is properly cited.

\begin{abstract}
We prove some quadruple random coincidence and quadruple random fixed point theorems under a set of conditions. We give examples to support our result. Our results are a generalization of the recent paper of Ćirić and Lakshmikantham (2009).
\end{abstract}

\section{Introduction}

Random nonlinear analysis is an important mathematical discipline which is mainly concerned with the study of random nonlinear operators and their properties and is needed for the study of various classes of random equations. Random fixed point theorems are stochastic generalizations of classical fixed point theorems. Random fixed point theorems for contraction mappings on separable complete metric spaces have been proved by several authors [1-12].

Existence of fixed points in partially ordered metric spaces was first investigated by Turinici [13], where he extended the Banach contraction principle in partially ordered sets. In 2004, Ran and Reurings [14] presented some applications of Turinici's theorem to matrix equations.

Bhaskar and Lakshmikantham [15] introduced the notion of a coupled fixed point and proved some coupled fixed point theorems for mappings satisfying a mixed monotone property. They discussed the problem of uniqueness of coupled fixed point and applied their theorems to problems of existence and uniqueness of solution for a periodic boundary value problem. Lakshmikantham and Ćirić [16] introduced the concept of mixed $g$-monotone mapping and proved coupled coincidence and coupled common fixed point theorems for commuting mappings, extending the theorems due to Bhaskar and Lakshmikantham [15]. Recently, Ćirić and Lakshmikantham [17] studied coupled random coincidence and coupled random fixed point theorems for a pair of random mappings $F: \Omega \times(X \times X) \rightarrow X$ and $g: \Omega \times$ $X \rightarrow X$, where $X$ is a complete separable metric space and $(\Omega, \Sigma)$ is a measurable space, under some contractive conditions. Very recently, Berinde and Borcut [18] introduced the concept of tripled fixed point and proved some related theorems. In a natural fashion, Karapinar and others [19-23] used the concept of quadruple fixed point and proved some fixed point theorems on the topic.

Following the above studies, we establish the existence and uniqueness of quadruple random coincidence and quadruple random fixed point theorems for a pair of random mappings which extend Theorems 2.2 and 2.3 of Ćirić and Lakshmikantham results [17].

\section{Preliminaries}

The concept of a mixed monotone property of the mapping $F: X \times X \rightarrow X$ has been introduced by Bhaskar and Lakshmikantham [15] by the following definitions.

Definition 1 (see [15]). Let $(X, \leq)$ be a partially ordered set and $F: X \times X \rightarrow X$. The map $F$ has the mixed monotone property if $F(x, y)$ is monotone nondecreasing in $x$ and is monotone nonincreasing in $y$; that is, for any $x, y \in X$,

$$
\begin{aligned}
& x_{1}, x_{2} \in X, \quad x_{1} \leq x_{2} \Longrightarrow F\left(x_{1}, y\right) \leq F\left(x_{2}, y\right), \\
& y_{1}, y_{2} \in X, \quad y_{1} \leq y_{2} \Longrightarrow F\left(x, y_{1}\right) \geq F\left(x, y_{2}\right) .
\end{aligned}
$$


Definition 2 (see [15]). An element $(x, y) \in X \times X$ is called a coupled fixed point of a mapping $F: X \times X \rightarrow X$ if

$$
F(x, y)=x, \quad F(y, x)=y .
$$

The concept of mixed monotone property is generalized to the concept of a mixed $g$-monotone property in [16].

Definition 3 (see [16]). Let $(X, \leq)$ be a partially ordered set and $F: X \times X \rightarrow X$ and $g: X \rightarrow X$. The mapping $F$ is said to have the mixed $g$-monotone property if $F(x, y)$ is monotone $g$-nondecreasing in $x$ and is monotone $g$-nonincreasing in $y$; that is, for any $x, y \in X$,

$$
\begin{aligned}
& x_{1}, x_{2} \in X, \quad g\left(x_{1}\right) \leq g\left(x_{2}\right) \Longrightarrow F\left(x_{1}, y\right) \leq F\left(x_{2}, y\right), \\
& y_{1}, y_{2} \in X, \quad g\left(y_{1}\right) \leq g\left(y_{2}\right) \Longrightarrow F\left(x, y_{1}\right) \geq F\left(x, y_{2}\right) .
\end{aligned}
$$

It is clear that if $g$ is the identity mapping, then Definition 3 reduces to Definition 1 .

Definition 4 (see [16]). An element $(x, y) \in X \times X$ is called a coupled coincidence point of a mapping $F: X \times X \rightarrow X$ and $g: X \rightarrow X$ if

$$
F(x, y)=g(x), \quad F(y, x)=g(y) .
$$

Berinde and Borcut [18] introduced the following partial order on the product space $X^{3}=X \times X \times X$ :

$$
(u, v, w) \leq(x, y, z)
$$

iff $x \geq u, \quad y \leq v, \quad$ only $z \geq w$,

where $(u, v, w),(x, y, z) \in X^{3}$. Regarding this partial order, we state the following definition.

Definition 5 (see [18]). Let $(X, \leq)$ be a partially ordered set and $F: X^{3} \rightarrow X$. The map $F$ has the mixed monotone property if $F(x, y, z)$ is monotone nondecreasing in $x$ and $z$ and is monotone nonincreasing in $y$; that is, for any $x, y, z \in$ $X$,

$$
\begin{aligned}
& x_{1}, x_{2} \in X, \quad x_{1} \leq x_{2} \Longrightarrow F(x, y, z) \leq F(x, y, z), \\
& y_{1}, y_{2} \in X, \quad y_{1} \leq y_{2} \Longrightarrow F\left(x, y_{1}, z\right) \geq F\left(x, y_{2}, z\right), \\
& z_{1}, z_{2} \in X, \quad z_{1} \leq z_{2} \Longrightarrow F\left(x, y, z_{1}\right) \leq F\left(x, y, z_{2}\right) .
\end{aligned}
$$

Definition 6 (see [18]). An element $(x, y, z) \in X^{3}$ is called a tripled fixed point of a mapping $F: X^{3} \rightarrow X$ if

$$
F(x, y, z)=x, \quad F(y, x, z)=y, \quad F(z, y, x)=z .
$$

Karapinar [19] introduced the concept of quadruple fixed point. He introduced the following partial order on the product space $X^{4}=X \times X \times X \times X$ :

$$
(u, v, w, s) \leq(x, y, z, t)
$$

iff $x \geq u, \quad y \leq v, \quad z \geq w, \quad$ only $t \leq s$,

where $(u, v, w, s),(x, y, z, t) \in X^{4}$. Regarding this partial order, we state the following definition.
Definition 7 (see [19]). Let $(X, \leq)$ be a partially ordered set and $F: X^{4} \rightarrow X$. The map $F$ has the mixed monotone property if $F(x, y, z, t)$ is monotone nondecreasing in $x$ and $z$ and is monotone nonincreasing in $y, t$; that is, for any $x, y, z, t \in X$,

$$
\begin{aligned}
& x_{1}, x_{2} \in X \text {, only } x_{1} \leq x_{2} \Longrightarrow F\left(x_{1}, y, z, t\right) \\
& \leq F\left(x_{2}, y, z, t\right), \\
& y_{1}, y_{2} \in X \text {, only } y_{1} \leq y_{2} \Longrightarrow F\left(x, y_{1}, z, t\right) \\
& \geq F\left(x, y_{2}, z, t\right), \\
& z_{1}, z_{2} \in X \text {, only } z_{1} \leq z_{2} \Longrightarrow F\left(x, y, z_{1}, t\right) \\
& \leq F\left(x, y, z_{2}, t\right), \\
& t_{1}, t_{2} \in X, \quad \text { only } t_{1} \leq t_{2} \Longrightarrow F\left(x, y, z, t_{1}\right) \\
& \geq F\left(x, y, z, t_{2}\right) \text {. }
\end{aligned}
$$

Definition 8 (see [19]). An element $(x, y, z, t) \in X^{4}$ is called a quadruple fixed point of a mapping $F: X^{4} \rightarrow X$ if

$$
\begin{array}{ll}
F(x, y, z, t)=x, & F(y, z, t, x)=y, \\
F(z, t, x, y)=z, & F(t, x, y, z)=t .
\end{array}
$$

Definition 9 (see [20]). Let $(X, \leq)$ be a partially ordered set and $F: X^{4} \rightarrow X$ and $g: X \rightarrow X$. The map $F$ has the mixed $g$-monotone property if $F(x, y, z, t)$ is monotone $g$ nondecreasing in $x$ and $z$ and is monotone $g$-nonincreasing in $y, t$; that is, for any $x, y, z, t \in X$,

$$
\begin{aligned}
x_{1}, x_{2} \in X, \quad g\left(x_{1}\right) & \leq g\left(x_{2}\right) \Longrightarrow F\left(x_{1}, y, z, t\right) \\
& \leq F\left(x_{2}, y, z, t\right), \\
y_{1}, y_{2} \in X, \quad g\left(y_{1}\right) & \leq g\left(y_{2}\right) \Longrightarrow F\left(x, y_{1}, z, t\right) \\
& \geq F\left(x, y_{2}, z, t\right), \\
z_{1}, z_{2} \in X, \quad g\left(z_{1}\right) & \leq g\left(z_{2}\right) \Longrightarrow F\left(x, y, z_{1}, t\right) \\
& \leq F\left(x, y, z_{2}, t\right), \\
t_{1}, t_{2} \in X, \quad g\left(t_{1}\right) & \leq g\left(t_{2}\right) \Longrightarrow F\left(x, y, z, t_{1}\right) \\
& \geq F\left(x, y, z, t_{2}\right) .
\end{aligned}
$$

Definition 10 (see [20]). An element $(x, y, z, t) \in X^{4}$ is called a quadruple coincidence point of a mapping $F: X^{4} \rightarrow X$ and $g: X \rightarrow X$ if

$$
\begin{array}{ll}
F(x, y, z, t)=g(x), & F(y, z, t, x)=g(y), \\
F(z, t, x, y)=g(z), & F(t, x, y, z)=g(t) .
\end{array}
$$

Definition 11 (see [20]). Let $F: X^{4} \rightarrow X$ and $g: X \rightarrow X$ be mappings. We say $F$ and $g$ are commutative if

$$
g(F(x, y, z, t))=F(g(x), g(y), g(z), g(t)),
$$

for all $x, y, z, t \in X$. 
Let $\Phi$ denote the all functions $\varphi:[0, \infty) \rightarrow[0, \infty)$ which are continuous and satisfy that

(i) $\varphi(t)<t$,

(ii) $\lim _{r \rightarrow t^{+}} \varphi(r)<t$ for each $t>0$.

Let $(\Omega, \Sigma)$ be a measurable space with $\Sigma$, a sigma algebra of subsets of $\Omega$, and let $(X, d)$ be a metric space. A mapping $T: \Omega \rightarrow X$ is called measurable if for any open subset $U$ of $X, T^{-1}(U)=\{w: T(w) \in U\} \in \Sigma$. A mapping $T: \Omega \times X \rightarrow X$ is said to be random mapping if for each fixed $x \in X$, the mapping $T(\cdot, x): \Omega \rightarrow X$ is measurable. A measurable mapping $\xi: \Omega \rightarrow X$ is called a random fixed point of the random mapping $T: \Omega \times X \rightarrow X$ if $T(w, \xi(w))=\xi(w)$ for each $w \in \Omega$. A measurable mapping $\xi: \Omega \rightarrow X$ is called a random coincidence of $T: \Omega \times X \rightarrow X$ and $g: \Omega \times X \rightarrow X$ if $T(w, \xi(w))=g(w, \xi(w))$ for each $w \in \Omega$.

Ćirić and Lakshmikantham in [17] proved coupled random fixed point theorems for a pair of random mappings $F: \Omega \times(X \times X) \rightarrow X$ and $g: \Omega \times X \rightarrow X$. They proved new results for random mixed $g$-monotone mappings, which extend the corresponding results for deterministic mixed monotone mappings of [16].

Theorem 12 (see [17]). Let $(X, d)$ be a complete separable metric space, let $(\Omega, \Sigma)$ be a measurable space, and let $F$ : $\Omega \times(X \times X) \rightarrow X$ and $g: \Omega \times X \rightarrow X$ be mappings such that

(1) $F(w, \cdot), g(w, \cdot)$ are continuous for all $w \in \Omega$,

(2) $F(\cdot, v), g(\cdot, x)$ are measurable for all $v \in X \times X$ and $x \in X$, respectively,

(3) $F: \Omega \times(X \times X) \rightarrow X$ and $g: \Omega \times X \rightarrow X$ are such that $F$ has the mixed $g$-monotone property and

$$
\begin{aligned}
& d(F(w,(x, y)), F(w,(u, v))) \\
& \quad \leq \varphi\left(\frac{d(g(w, x), g(w, u))+d(g(w, y), g(w, v))}{2}\right),
\end{aligned}
$$

for all $x, y, u, v \in X$ for which $g(w, x) \leq g(w, u)$ and $g(w, y) \geq$ $g(w, v)$ for all $w \in \Omega$. Suppose $g(w \times X)=X$ for each $w \in \Omega$ and $g$ is continuous and commutes with $F$ and also suppose either

(a) $F$ is continuous or

(b) $X$ has the following property:

(1) if a nondecreasing sequence $\left\{x_{n}\right\} \rightarrow x$ then $x_{n} \leq$ $x$ for all $n$,

(2) if a nonincreasing sequence $\left\{y_{n}\right\} \rightarrow y$ then $y_{n} \geq$ $y$ for all $n$.

If there exist measurable mappings $\xi_{0}, \eta_{0}: \Omega \rightarrow X$ such that

$$
\begin{gathered}
g\left(w, \xi_{0}(w)\right) \leq F\left(w,\left(\xi_{0}(w), \eta_{0}(w)\right)\right), \\
g\left(w, \eta_{0}(w)\right) \geq F\left(w,\left(\eta_{0}(w), \xi_{0}(w)\right)\right)
\end{gathered}
$$

for all $w \in \Omega$, then there are measurable mappings $\zeta, \theta: \Omega \rightarrow$ $X$ such that

$$
\begin{aligned}
& F(w,(\zeta(w), \theta(w)))=g(w, \zeta(w)), \\
& F(w,(\theta(w), \zeta(w)))=g(w, \theta(w)),
\end{aligned}
$$

for all $w \in \Omega$; that is, $F$ and $g$ have a coupled random coincidence.

\section{Main Results}

The following theorem is our main result.

Theorem 13. Let $(X, d)$ be a complete separable metric space, and let $(\Omega, \Sigma)$ be a measurable space and $\varphi \in \Phi$. Let $F: \Omega \times$ $X^{4} \rightarrow X$ and $g: \Omega \times X \rightarrow X$ be mappings such that

(1) $F(w, \cdot), g(w, \cdot)$ are continuous for all $w \in \Omega$,

(2) $F(\cdot, u), g(\cdot, v)$ are measurable for all $u \in X^{4}$ and $v \in X$, respectively,

(3) $F: \Omega \times X^{4} \rightarrow X$ and $g: \Omega \times X \rightarrow X$ are such that $F$ has the mixed $g$-monotone property and

$$
\begin{aligned}
& d(F(w,(x, y, z, s)), F(w,(u, v, r, t))) \\
& \leq \varphi((d(g(w, x), g(w, u))+d(g(w, y), g(w, v)) \\
& \quad+d(g(w, z), g(w, r)) \\
& \left.\quad+d(g(w, s), g(w, t))) \times(4)^{-1}\right),
\end{aligned}
$$

for all $x, y, z, s, u, v, r, t \in X$ for which $g(w, x) \leq g(w, u)$, $g(w, y) \geq g(w, v), g(w, z) \leq g(w, r)$, and $g(w, s) \geq g(w, t)$ for all $w \in \Omega$. Suppose $g(w \times X)=X$ for each $w \in \Omega$ and $g$ is continuous and commutes with $F$ and also suppose either

(a) F is continuous or

(b) X has the following property:

(1) if a nondecreasing sequence $\left\{x_{n}\right\} \rightarrow x$ then $x_{n} \leq$ $x$ for all $n$,

(2) if a nonincreasing sequence $\left\{y_{n}\right\} \rightarrow y$ then $y_{n} \geq$ $y$ for all $n$.

If there exist measurable mappings $\xi_{0}, \eta_{0}, \zeta_{0}, \rho_{0}: \Omega \rightarrow X$ such that

$$
\begin{array}{r}
g\left(w, \xi_{0}(w)\right) \leq F\left(w,\left(\xi_{0}(w), \eta_{0}(w), \zeta_{0}(w), \rho_{0}(w)\right)\right), \\
g\left(w, \eta_{0}(w)\right) \geq F\left(w,\left(\eta_{0}(w), \zeta_{0}(w), \rho_{0}(w), \xi_{0}(w)\right)\right), \\
g\left(w, \zeta_{0}(w)\right) \leq F\left(w,\left(\zeta_{0}(w), \rho_{0}(w), \xi_{0}(w), \eta_{0}(w)\right)\right), \\
g\left(w, \rho_{0}(w)\right) \geq F\left(w,\left(\rho_{0}(w), \xi_{0}(w), \eta_{0}(w), \zeta_{0}(w)\right)\right), \\
\text { for all } w \in \Omega,
\end{array}
$$


then there are measurable mappings $\xi, \eta, \zeta, \rho: \Omega \rightarrow X$ such that

$$
\begin{aligned}
& F(w,(\xi(w), \eta(w), \zeta(w), \rho(w)))=g(w, \xi(w)), \\
& F(w,(\eta(w), \zeta(w), \rho(w), \xi(w)))=g(w, \eta(w)), \\
& F(w,(\zeta(w), \rho(w), \xi(w), \eta(w)))=g(w, \zeta(w)), \\
& F(w,(\rho(w), \xi(w), \eta(w), \zeta(w)))=g(w, \rho(w)),
\end{aligned}
$$

for all $w \in \Omega$; that is, $F$ and $g$ have a quadruple random coincidence.

Proof. Let $\Theta=\{\xi: \Omega \rightarrow X\}$ be a family of measurable mappings. Define a function $h: \Omega \times X \rightarrow R^{+}$as follows: $h(w, x)=d(x, g(w, x))$. Since $x \rightarrow g(w, x)$ is continuous for all $w \in \Omega$, we conclude that $h(w, \cdot)$ is continuous for all $w \in \Omega$. Also, since $w \rightarrow g(w, x)$ is measurable for all $x \in X$, we conclude that $h(w, \cdot)$ is measurable for all $w \in \Omega$ (see Wagner [11], page 868). Thus, $h(w, x)$ is the Caratheodory function. Therefore, if $\xi: \Omega \rightarrow X$ is a measurable mapping, then $w \rightarrow$ $h(w, \xi(w))$ is also measurable (see [9]). Also, for each $\xi \in \Theta$, the function $\eta: \Omega \rightarrow X$ defined by $\eta(w)=g(w, \xi(w))$ is measurable; that is, $\eta \in \Theta$.

Now, we will construct four sequences of measurable mappings $\left\{\xi_{n}\right\},\left\{\eta_{n}\right\},\left\{\zeta_{n}\right\}$, and $\left\{\rho_{n}\right\}$ in $\Theta$ and four sequences $\left\{g\left(w, \xi_{n}(w)\right)\right\},\left\{g\left(w, \eta_{n}(w)\right)\right\},\left\{g\left(w, \zeta_{n}(w)\right)\right\}$, and $\left\{g\left(w, \rho_{n}(w)\right)\right\}$ in $X$ as follows: by assumption (18), let $\xi_{0}, \eta_{0}, \zeta_{0}, \rho_{0} \in \Theta$ such that

$$
\begin{aligned}
& g\left(w, \xi_{0}(w)\right) \leq F\left(w,\left(\xi_{0}(w), \eta_{0}(w), \zeta_{0}(w), \rho_{0}(w)\right)\right), \\
& g\left(w, \eta_{0}(w)\right) \geq F\left(w,\left(\eta_{0}(w), \zeta_{0}(w), \rho_{0}(w), \xi_{0}(w)\right)\right), \\
& g\left(w, \zeta_{0}(w)\right) \leq F\left(w,\left(\zeta_{0}(w), \rho_{0}(w), \xi_{0}(w), \eta_{0}(w)\right)\right), \\
& g\left(w, \rho_{0}(w)\right) \geq F\left(w,\left(\rho_{0}(w), \xi_{0}(w), \eta_{0}(w), \zeta_{0}(w)\right)\right),
\end{aligned}
$$

for all $w \in \Omega$. Since $F\left(w \times X^{4}\right) \in X=g(w \times X)$, then by a sort of Filippov measurable implicit function theorem $[1,5,6,24]$, we can choose $\xi_{1}, \eta_{1}, \zeta_{1}, \rho_{1} \in \Theta$ such that

$$
\begin{aligned}
& g\left(w, \xi_{1}(w)\right)=F\left(w,\left(\xi_{0}(w), \eta_{0}(w), \zeta_{0}(w), \rho_{0}(w)\right)\right), \\
& g\left(w, \eta_{1}(w)\right)=F\left(w,\left(\eta_{0}(w), \zeta_{0}(w), \rho_{0}(w), \xi_{0}(w)\right)\right), \\
& g\left(w, \zeta_{1}(w)\right)=F\left(w,\left(\zeta_{0}(w), \rho_{0}(w), \xi_{0}(w), \eta_{0}(w)\right)\right), \\
& g\left(w, \rho_{1}(w)\right)=F\left(w,\left(\rho_{0}(w), \xi_{0}(w), \eta_{0}(w), \zeta_{0}(w)\right)\right),
\end{aligned}
$$

Again taking into account that $F\left(w \times X^{4}\right) \in X=g(w \times X)$ and continuing this process, we can construct sequences $\left\{\xi_{n}\right\}$, $\left\{\eta_{n}\right\},\left\{\zeta_{n}\right\}$, and $\left\{\rho_{n}\right\}$ in $X$ such that

$$
\begin{aligned}
& g\left(w, \xi_{n+1}(w)\right)=F\left(w,\left(\xi_{n}(w), \eta_{n}(w), \zeta_{n}(w), \rho_{n}(w)\right)\right), \\
& g\left(w, \eta_{n+1}(w)\right)=F\left(w,\left(\eta_{n}(w), \zeta_{n}(w), \rho_{n}(w), \xi_{n}(w)\right)\right), \\
& g\left(w, \zeta_{n+1}(w)\right)=F\left(w,\left(\zeta_{n}(w), \rho_{n}(w), \xi_{n}(w), \eta_{n}(w)\right)\right), \\
& g\left(w, \rho_{n+1}(w)\right)=F\left(w,\left(\rho_{n}(w), \xi_{n}(w), \eta_{n}(w), \zeta_{n}(w)\right)\right),
\end{aligned}
$$$$
\text { for all } w \in \Omega \text {. }
$$

We claim for all $n \geq 0$ that

$$
\begin{aligned}
& g\left(w, \xi_{n}(w)\right) \leq g\left(w, \xi_{n+1}(w)\right), \\
& g\left(w, \eta_{n}(w)\right) \geq g\left(w, \eta_{n+1}(w)\right), \\
& g\left(w, \zeta_{n}(w)\right) \leq g\left(w, \zeta_{n+1}(w)\right), \\
& g\left(w, \rho_{n}(w)\right) \geq g\left(w, \rho_{n+1}(w)\right) .
\end{aligned}
$$

Indeed, we will use mathematical induction. By using (18) and (21) together, we obtain

$$
\begin{aligned}
g\left(w, \xi_{0}(w)\right) & \leq F\left(w,\left(\xi_{0}(w), \eta_{0}(w), \zeta_{0}(w), \rho_{0}(w)\right)\right) \\
& =g\left(w, \xi_{1}(w)\right), \\
g\left(w, \eta_{0}(w)\right) & \geq F\left(w,\left(\eta_{0}(w), \zeta_{0}(w), \rho_{0}(w), \xi_{0}(w)\right)\right) \\
& =g\left(w, \eta_{1}(w)\right), \\
g\left(w, \zeta_{0}(w)\right) & \leq F\left(w,\left(\zeta_{0}(w), \rho_{0}(w), \xi_{0}(w), \eta_{0}(w)\right)\right) \\
& =g\left(w, \zeta_{1}(w)\right), \\
g\left(w, \rho_{0}(w)\right) & \geq F\left(w,\left(\rho_{0}(w), \xi_{0}(w), \eta_{0}(w), \zeta_{0}(w)\right)\right) \\
& =g\left(w, \rho_{1}(w)\right),
\end{aligned}
$$

for all $w \in \Omega$. Therefore (23) holds for $n=0$.

Suppose that (23) holds for some $n \geq 0$. Then, since $F$ has the mixed $g$-monotone property and by $(22)$ we have

$$
\begin{aligned}
g( & \left.w, \xi_{n+1}(w)\right) \\
& =F\left(w,\left(\xi_{n}(w), \eta_{n}(w), \zeta_{n}(w), \rho_{n}(w)\right)\right) \\
& \leq F\left(w,\left(\xi_{n+1}(w), \eta_{n}(w), \zeta_{n}(w), \rho_{n}(w)\right)\right) \\
& \leq F\left(w,\left(\xi_{n+1}(w), \eta_{n}(w), \zeta_{n+1}(w), \rho_{n}(w)\right)\right) \\
& \leq F\left(w,\left(\xi_{n+1}(w), \eta_{n+1}(w), \zeta_{n+1}(w), \rho_{n}(w)\right)\right) \\
& \leq F\left(w,\left(\xi_{n+1}(w), \eta_{n+1}(w), \zeta_{n+1}(w), \rho_{n+1}(w)\right)\right) \\
& =g\left(w, \xi_{n+2}(w)\right)
\end{aligned}
$$




$$
\begin{aligned}
& g\left(w, \eta_{n+2}(w)\right) \\
& =F\left(w,\left(\eta_{n+1}(w), \zeta_{n+1}(w), \rho_{n+1}(w), \xi_{n+1}(w)\right)\right) \\
& \leq F\left(w,\left(\eta_{n+1}(w), \zeta_{n}(w), \rho_{n+1}(w), \xi_{n+1}(w)\right)\right) \\
& \leq F\left(w,\left(\eta_{n}(w), \zeta_{n}(w), \rho_{n+1}(w), \xi_{n+1}(w)\right)\right) \\
& \leq F\left(w,\left(\eta_{n}(w), \zeta_{n}(w), \rho_{n+1}(w), \xi_{n}(w)\right)\right) \\
& \leq F\left(w,\left(\eta_{n}(w), \zeta_{n}(w), \rho_{n}(w), \xi_{n}(w)\right)\right) \\
& =g\left(w, \eta_{n+1}(w)\right) \text {, } \\
& g\left(w, \zeta_{n+1}(w)\right) \\
& =F\left(w,\left(\zeta_{n}(w), \rho_{n}(w), \xi_{n}(w), \eta_{n}(w)\right)\right) \\
& \leq F\left(w,\left(\zeta_{n+1}(w), \rho_{n}(w), \xi_{n}(w), \eta_{n}(w)\right)\right) \\
& \leq F\left(w,\left(\zeta_{n+1}(w), \rho_{n+1}(w), \xi_{n}(w), \eta_{n}(w)\right)\right) \\
& \leq F\left(w,\left(\zeta_{n+1}(w), \rho_{n+1}(w), \xi_{n+1}(w), \eta_{n}(w)\right)\right) \\
& \leq F\left(w,\left(\zeta_{n+1}(w), \rho_{n+1}(w), \xi_{n+1}(w), \eta_{n+1}(w)\right)\right) \\
& =g\left(w, \zeta_{n+2}(w)\right) \text {, } \\
& g\left(w, \rho_{n+2}(w)\right) \\
& =F\left(w,\left(\rho_{n+1}(w), \xi_{n+1}(w), \eta_{n+1}(w), \zeta_{n+1}(w)\right)\right) \\
& \leq F\left(w,\left(\rho_{n+1}(w), \xi_{n}(w), \eta_{n+1}(w), \zeta_{n+1}(w)\right)\right) \\
& \leq F\left(w,\left(\rho_{n}(w), \xi_{n}(w), \eta_{n+1}(w), \zeta_{n+1}(w)\right)\right) \\
& \leq F\left(w,\left(\rho_{n}(w), \xi_{n}(w), \eta_{n+1}(w), \zeta_{n}(w)\right)\right) \\
& \leq F\left(w,\left(\rho_{n}(w), \xi_{n}(w), \eta_{n}(w), \zeta_{n}(w)\right)\right) \\
& =g\left(w, \rho_{n+1}(w)\right) \text {. }
\end{aligned}
$$

Thus, (23) holds for all $n \geq 0$.

Denote

$$
\begin{aligned}
\delta_{n}= & d\left(g\left(w, \xi_{n}(w)\right), g\left(w, \xi_{n+1}(w)\right)\right) \\
& +d\left(g\left(w, \eta_{n}(w)\right), g\left(w, \eta_{n+1}(w)\right)\right) \\
& +d\left(g\left(w, \zeta_{n}(w)\right), g\left(w, \zeta_{n+1}(w)\right)\right) \\
& +d\left(g\left(w, \rho_{n}(w)\right), g\left(w, \rho_{n+1}(w)\right)\right) .
\end{aligned}
$$

We show that

$$
\frac{\delta_{n+1}}{4} \leq \varphi\left(\frac{\delta_{n}}{4}\right) \text { for all } n \geq 0
$$

Due to (17), (22), and (23)

$$
\begin{aligned}
& d\left(g\left(w, \xi_{n+1}(w)\right), g\left(w, \xi_{n+2}(w)\right)\right) \\
& =d\left(F\left(w,\left(\xi_{n}(w), \eta_{n}(w), \zeta_{n}(w), \rho_{n}(w)\right)\right),\right. \\
& \left.F\left(w,\left(\xi_{n+1}(w), \eta_{n+1}(w), \zeta_{n+1}(w), \rho_{n+1}(w)\right)\right)\right) \\
& \leq \varphi\left(\frac { 1 } { 4 } \left[d\left(g\left(w, \xi_{n}(w)\right), g\left(w, \xi_{n+1}(w)\right)\right)\right.\right. \\
& +d\left(g\left(w, \eta_{n}(w)\right), g\left(w, \eta_{n+1}(w)\right)\right) \\
& +d\left(g\left(w, \zeta_{n}(w)\right), g\left(w, \zeta_{n+1}(w)\right)\right) \\
& \left.\left.+d\left(g\left(w, \rho_{n}(w)\right), g\left(w, \rho_{n+1}(w)\right)\right)\right]\right) \\
& =\varphi\left(\frac{\delta_{n}}{4}\right) .
\end{aligned}
$$

Similarly,

$$
\begin{aligned}
& d\left(g\left(w, \eta_{n+1}(w)\right), g\left(w, \eta_{n+2}(w)\right)\right) \\
& =d\left(F\left(w,\left(\eta_{n}(w), \zeta_{n}(w), \rho_{n}(w), \xi_{n}(w)\right)\right),\right. \\
& \left.\quad F\left(w,\left(\eta_{n+1}(w), \zeta_{n+1}(w), \rho_{n+1}(w), \xi_{n+1}(w)\right)\right)\right) \\
& =\varphi\left(\frac{\delta_{n}}{4}\right), \\
& d\left(g\left(w, \zeta_{n+1}(w)\right), g\left(w, \zeta_{n+2}(w)\right)\right) \\
& =d\left(F\left(w,\left(\zeta_{n}(w), \rho_{n}(w), \xi_{n}(w), \eta_{n}(w)\right)\right),\right. \\
& \left.\quad F\left(w,\left(\zeta_{n+1}(w), \rho_{n+1}(w), \xi_{n+1}(w), \eta_{n+1}(w)\right)\right)\right) \\
& =\varphi\left(\frac{\delta_{n}}{4}\right), \\
& d\left(g\left(w, \rho_{n+1}(w)\right), g\left(w, \rho_{n+2}(w)\right)\right) \\
& =d\left(F\left(w,\left(\rho_{n}(w), \xi_{n}(w), \eta_{n}(w), \zeta_{n}(w)\right)\right),\right. \\
& \left.F\left(w,\left(\rho_{n+1}(w), \xi_{n+1}(w), \eta_{n+1}(w), \zeta_{n+1}(w)\right)\right)\right) \\
& =\varphi\left(\frac{\delta_{n}}{4}\right) .
\end{aligned}
$$

Summing up (28)-(31) and dividing by 4 , we get (27). Since $\varphi(t)<t$ for all $t>0$, then $\delta_{n+1} \leq \delta_{n}$ for all $n$; that is, $\left\{\delta_{n}\right\}$ is the monotone decreasing sequence of positive reals and so there is some $\delta \geq 0$ such that

$$
\lim _{n \rightarrow \infty} \delta_{n}=\delta+
$$


We will prove that $\delta=0$. Suppose, to the contrary, that $\delta>0$. Taking the limit in (27) as $\delta_{n} \rightarrow \delta+$ and using the assumption that $\lim _{r \rightarrow t^{+}} \varphi(r)<t$ for each $t>0$, we obtain

$$
\frac{\delta}{4}=\lim _{n \rightarrow \infty} \frac{\delta_{n+1}}{4} \leq \lim _{n \rightarrow \infty} \varphi\left(\frac{\delta_{n}}{4}\right)=\lim _{\delta_{n} \rightarrow \delta+} \varphi\left(\frac{\delta_{n}}{4}\right)<\frac{\delta}{4},
$$

which is a contradiction; thus $\delta=0$.

Now, we will prove that $\left\{g\left(w, \xi_{n}(w)\right)\right\},\left\{g\left(w, \eta_{n}(w)\right)\right\}$, $\left\{g\left(w, \zeta_{n}(w)\right)\right\}$, and $\left\{g\left(w, \rho_{n}(w)\right)\right\}$ are Cauchy sequences. Suppose, to the contrary, that at least one of $\left\{g\left(w, \xi_{n}(w)\right)\right\}$, $\left\{g\left(w, \eta_{n}(w)\right)\right\},\left\{g\left(w, \zeta_{n}(w)\right)\right\}$, or $\left\{g\left(w, \rho_{n}(w)\right)\right\}$ is not Cauchy sequence. Then, there exists an $\varepsilon>0$ for which we can find subsequences of positive integers $\{m(k)\},\{n(k)\}$ with $n(k)>$ $m(k) \geq k$ such that

$$
\begin{aligned}
r_{k}=[ & d\left(g\left(w, \xi_{m(k)}(w)\right), g\left(w, \xi_{n(k)}(w)\right)\right) \\
& +d\left(g\left(w, \eta_{m(k)}(w)\right), g\left(w, \eta_{n(k)}(w)\right)\right) \\
& +d\left(g\left(w, \zeta_{m(k)}(w)\right), g\left(w, \zeta_{n(k)}(w)\right)\right) \\
& \left.+d\left(g\left(w, \rho_{m(k)}(w)\right), g\left(w, \rho_{n(k)}(w)\right)\right)\right] \geq \varepsilon .
\end{aligned}
$$

Further, corresponding to $m(k)$, we can choose $n(k)$ such that it is the smallest integer with $n(k)>m(k)$ and satisfying (34). Then

$$
\begin{aligned}
& {\left[d\left(g\left(w, \xi_{m(k)}(w)\right), g\left(w, \xi_{n(k)-1}(w)\right)\right)\right.} \\
& +d\left(g\left(w, \eta_{m(k)}(w)\right), g\left(w, \eta_{n(k)-1}(w)\right)\right) \\
& +d\left(g\left(w, \zeta_{m(k)}(w)\right), g\left(w, \zeta_{n(k)-1}(w)\right)\right) \\
& \left.+d\left(g\left(w, \rho_{m(k)}(w)\right), g\left(w, \rho_{n(k)-1}(w)\right)\right)\right]<\varepsilon .
\end{aligned}
$$

By triangle inequality with (34) and (35), we get

$$
\begin{aligned}
\varepsilon \leq r_{k} & \\
= & {\left[d\left(g\left(w, \xi_{m(k)}(w)\right), g\left(w, \xi_{n(k)}(w)\right)\right)\right.} \\
& +d\left(g\left(w, \eta_{m(k)}(w)\right), g\left(w, \eta_{n(k)}(w)\right)\right) \\
& +d\left(g\left(w, \zeta_{m(k)}(w)\right), g\left(w, \zeta_{n(k)}(w)\right)\right) \\
& \left.+d\left(g\left(w, \rho_{m(k)}(w)\right), g\left(w, \rho_{n(k)}(w)\right)\right)\right] \\
\leq & d\left(g\left(w, \xi_{m(k)}(w)\right), g\left(w, \xi_{n(k)-1}(w)\right)\right) \\
& +d\left(g\left(w, \xi_{n(k)-1}(w)\right), g\left(w, \xi_{n(k)}(w)\right)\right) \\
& +d\left(g\left(w, \eta_{m(k)}(w)\right), g\left(w, \eta_{n(k)-1}(w)\right)\right) \\
& +d\left(g\left(w, \eta_{n(k)-1}(w)\right), g\left(w, \eta_{n(k)}(w)\right)\right)
\end{aligned}
$$

$$
\begin{aligned}
+ & d\left(g\left(w, \zeta_{m(k)}(w)\right), g\left(w, \zeta_{n(k)-1}(w)\right)\right) \\
+ & d\left(g\left(w, \zeta_{n(k)-1}(w)\right), g\left(w, \zeta_{n(k)}(w)\right)\right) \\
+ & d\left(g\left(w, \rho_{m(k)}(w)\right), g\left(w, \rho_{n(k)-1}(w)\right)\right) \\
+ & d\left(g\left(w, \rho_{n(k)-1}(w)\right), g\left(w, \rho_{n(k)}(w)\right)\right) \\
= & d\left(g\left(w, \xi_{m(k)}(w)\right), g\left(w, \xi_{n(k)-1}(w)\right)\right) \\
+ & d\left(g\left(w, \eta_{m(k)}(w)\right), g\left(w, \eta_{n(k)-1}(w)\right)\right) \\
+ & d\left(g\left(w, \zeta_{m(k)}(w)\right), g\left(w, \zeta_{n(k)-1}(w)\right)\right) \\
+ & \left.d\left(g\left(w, \rho_{m(k)}(w)\right), g\left(w, \rho_{n(k)-1}(w)\right)\right)\right] \\
+ & {\left[d\left(g\left(w, \xi_{n(k)-1}(w)\right), g\left(w, \xi_{n(k)}(w)\right)\right)\right.} \\
& +d\left(g\left(w, \eta_{n(k)-1}(w)\right), g\left(w, \eta_{n(k)}(w)\right)\right) \\
+ & d\left(g\left(w, \zeta_{n(k)-1}(w)\right), g\left(w, \zeta_{n(k)}(w)\right)\right) \\
+ & \left.+d\left(g\left(w, \rho_{n(k)-1}(w)\right), g\left(w, \rho_{n(k)}(w)\right)\right)\right] \\
\varepsilon+ & \delta_{n(k)-1} \cdot
\end{aligned}
$$

Letting $k \rightarrow \infty$ in (36), we get

$$
\lim _{k \rightarrow \infty} r_{k}=\varepsilon+
$$

Again using triangle inequality,

$$
\begin{aligned}
r_{k}=[ & d\left(g\left(w, \xi_{m(k)}(w)\right), g\left(w, \xi_{n(k)}(w)\right)\right) \\
& +d\left(g\left(w, \eta_{m(k)}(w)\right), g\left(w, \eta_{n(k)}(w)\right)\right) \\
& +d\left(g\left(w, \zeta_{m(k)}(w)\right), g\left(w, \zeta_{n(k)}(w)\right)\right) \\
& \left.+d\left(g\left(w, \rho_{m(k)}(w)\right), g\left(w, \rho_{n(k)}(w)\right)\right)\right] \\
\leq & d\left(g\left(w, \xi_{m(k)}(w)\right), g\left(w, \xi_{m(k)+1}(w)\right)\right) \\
& +d\left(g\left(w, \xi_{m(k)+1}(w)\right), g\left(w, \xi_{n(k)+1}(w)\right)\right) \\
& +d\left(g\left(w, \xi_{n(k)+1}(w)\right), g\left(w, \xi_{n(k)}(w)\right)\right) \\
& +d\left(g\left(w, \eta_{m(k)}(w)\right), g\left(w, \eta_{m(k)+1}(w)\right)\right) \\
& +d\left(g\left(w, \eta_{m(k)+1}(w)\right), g\left(w, \eta_{n(k)+1}(w)\right)\right) \\
& +d\left(g\left(w, \eta_{n(k)+1}(w)\right), g\left(w, \eta_{n(k)}(w)\right)\right) \\
& +d\left(g\left(w, \zeta_{m(k)}(w)\right), g\left(w, \zeta_{m(k)+1}(w)\right)\right) \\
& +d\left(g\left(w, \zeta_{m(k)+1}(w)\right), g\left(w, \zeta_{n(k)+1}(w)\right)\right) \\
& +d\left(g\left(w, \zeta_{n(k)+1}(w)\right), g\left(w, \zeta_{n(k)}(w)\right)\right) \\
+ & +d\left(g\left(w, \rho_{m(k)}(w)\right), g\left(w, \rho_{m(k)+1}(w)\right)\right) \\
& \left.+d\left(w, \rho_{m(k)+1}(w)\right), g\left(w, \rho_{n(k)+1}(w)\right)\right) \\
& \left.\left.+\rho_{n(k)+1}(w)\right), g\left(w, \rho_{n(k)}(w)\right)\right)
\end{aligned}
$$




$$
\begin{aligned}
\leq & \delta_{m(k)}+\delta_{n(k)} \\
& +d\left(g\left(w, \xi_{m(k)+1}(w)\right), g\left(w, \xi_{n(k)+1}(w)\right)\right) \\
& +d\left(g\left(w, \eta_{m(k)+1}(w)\right), g\left(w, \eta_{n(k)+1}(w)\right)\right) \\
& +d\left(g\left(w, \zeta_{m(k)+1}(w)\right), g\left(w, \zeta_{n(k)+1}(w)\right)\right) \\
& +d\left(g\left(w, \rho_{m(k)+1}(w)\right), g\left(w, \rho_{n(k)+1}(w)\right)\right) .
\end{aligned}
$$

Since $n(k)>m(k)$, then from (23), we have

$$
\begin{aligned}
& g\left(w, \xi_{n(k)}(w)\right) \geq g\left(w, \xi_{m(k)}(w)\right), \\
& g\left(w, \eta_{n(k)}(w)\right) \leq g\left(w, \eta_{m(k)}(w)\right), \\
& g\left(w, \zeta_{n(k)}(w)\right) \geq g\left(w, \zeta_{m(k)}(w)\right), \\
& g\left(w, \rho_{n(k)}(w)\right) \leq g\left(w, \rho_{m(k)}(w)\right) .
\end{aligned}
$$

Hence from (17) and (22), we obtain

$$
\begin{gathered}
d\left(g\left(w, \xi_{m(k)+1}(w)\right), g\left(w, \xi_{n(k)+1}(w)\right)\right) \\
=d\left(F\left(w,\left(\xi_{m(k)}(w), \eta_{m(k)}(w), \zeta_{m(k)}(w), \rho_{m(k)}(w)\right)\right),\right. \\
\left.F\left(w,\left(\xi_{n(k)}(w), \eta_{n(k)}(w), \zeta_{n(k)}(w), \rho_{n(k)}(w)\right)\right)\right) \\
\leq \varphi\left(\frac { 1 } { 4 } \left[d\left(g\left(w, \xi_{m(k)}(w)\right), g\left(w, \xi_{n(k)}(w)\right)\right)\right.\right. \\
+d\left(g\left(w, \eta_{m(k)}(w)\right), g\left(w, \eta_{n(k)}(w)\right)\right) \\
+d\left(g\left(w, \zeta_{m(k)}(w)\right), g\left(w, \zeta_{n(k)}(w)\right)\right) \\
\left.\left.\quad+d\left(g\left(w, \rho_{m(k)}(w)\right), g\left(w, \rho_{n(k)}(w)\right)\right)\right]\right) \\
=\varphi\left(\frac{r_{k}}{4}\right) .
\end{gathered}
$$

By the same way, we have

$$
\begin{aligned}
& d\left(g\left(w, \eta_{m(k)+1}(w)\right), g\left(w, \eta_{n(k)+1}(w)\right)\right) \\
& =d\left(F\left(w,\left(\eta_{m(k)}(w), \zeta_{m(k)}(w), \rho_{m(k)}(w), \xi_{m(k)}(w)\right)\right),\right. \\
& \left.F\left(w,\left(\eta_{n(k)}(w), \zeta_{n(k)}(w), \rho_{n(k)}(w), \xi_{n(k)}(w)\right)\right)\right) \\
& \leq \varphi\left(\frac { 1 } { 4 } \left[d\left(g\left(w, \eta_{m(k)}(w)\right), g\left(w, \eta_{n(k)}(w)\right)\right)\right.\right. \\
& +d\left(g\left(w, \zeta_{m(k)}(w)\right), g\left(w, \zeta_{n(k)}(w)\right)\right)
\end{aligned}
$$

$$
\begin{aligned}
& +d\left(g\left(w, \rho_{m(k)}(w)\right), g\left(w, \rho_{n(k)}(w)\right)\right) \\
& \left.\left.+d\left(g\left(w, \xi_{m(k)}(w)\right), g\left(w, \xi_{n(k)}(w)\right)\right)\right]\right) \\
& =\varphi\left(\frac{r_{k}}{4}\right),
\end{aligned}
$$$$
d\left(g\left(w, \zeta_{m(k)+1}(w)\right), g\left(w, \zeta_{n(k)+1}(w)\right)\right)
$$$$
=d\left(F\left(w,\left(\zeta_{m(k)}(w), \rho_{m(k)}(w), \xi_{m(k)}(w), \eta_{m(k)}(w)\right)\right),\right.
$$$$
\left.F\left(w,\left(\zeta_{n(k)}(w), \rho_{n(k)}(w), \xi_{n(k)}(w), \eta_{n(k)}(w)\right)\right)\right)
$$$$
\leq \varphi\left(\frac { 1 } { 4 } \left[d\left(g\left(w, \zeta_{m(k)}(w)\right), g\left(w, \zeta_{n(k)}(w)\right)\right)\right.\right.
$$$$
+d\left(g\left(w, \rho_{m(k)}(w)\right), g\left(w, \rho_{n(k)}(w)\right)\right)
$$$$
+d\left(g\left(w, \xi_{m(k)}(w)\right), g\left(w, \xi_{n(k)}(w)\right)\right)
$$$$
\left.\left.+d\left(g\left(w, \eta_{m(k)}(w)\right), g\left(w, \eta_{n(k)}(w)\right)\right)\right]\right)
$$$$
=\varphi\left(\frac{r_{k}}{4}\right)
$$

$$
\begin{gathered}
d\left(g\left(w, \rho_{m(k)+1}(w)\right), g\left(w, \rho_{n(k)+1}(w)\right)\right) \\
=d\left(F\left(w,\left(\rho_{m(k)}(w), \xi_{m(k)}(w), \eta_{m(k)}(w), \zeta_{m(k)}(w)\right)\right)\right. \\
\left.F\left(w,\left(\rho_{n(k)}(w), \xi_{n(k)}(w), \eta_{n(k)}(w), \zeta_{n(k)}(w)\right)\right)\right) \\
\leq \varphi\left(\frac { 1 } { 4 } \left[d\left(g\left(w, \rho_{m(k)}(w)\right), g\left(w, \rho_{n(k)}(w)\right)\right)\right.\right. \\
+d\left(g\left(w, \xi_{m(k)}(w)\right), g\left(w, \xi_{n(k)}(w)\right)\right) \\
+d\left(g\left(w, \eta_{m(k)}(w)\right), g\left(w, \eta_{n(k)}(w)\right)\right) \\
\left.\left.\quad+d\left(g\left(w, \zeta_{m(k)}(w)\right), g\left(w, \zeta_{n(k)}(w)\right)\right)\right]\right) \\
=\varphi\left(\frac{r_{k}}{4}\right) .
\end{gathered}
$$

Inserting (40)-(43) in (38), we get

$$
r_{k} \leq \delta_{m(k)}+\delta_{n(k)}+4 \varphi\left(\frac{r_{k}}{4}\right)
$$

Letting $k \rightarrow \infty$, we get

$$
\varepsilon \leq 4 \lim _{k \rightarrow \infty} \varphi\left(\frac{r_{k}}{4}\right)=4 \lim _{r_{k} \rightarrow \varepsilon+} \varphi\left(\frac{r_{k}}{4}\right)<4 \frac{\varepsilon}{4}=\varepsilon
$$

a contradiction. This shows that $\left\{g\left(w, \xi_{n}(w)\right)\right\},\left\{g\left(w, \eta_{n}(w)\right)\right\}$, $\left\{g\left(w, \zeta_{n}(w)\right)\right\}$, and $\left\{g\left(w, \rho_{n}(w)\right)\right\}$ are Cauchy sequences. Since 
$X$ is complete and $g(w \times X)=X$, then there exist $\theta_{0}, \vartheta_{0}, \mu_{0}, v_{0} \in \Theta$ such that

$$
\begin{aligned}
& \lim _{n \rightarrow \infty} g\left(w, \xi_{n}(w)\right)=g\left(w, \theta_{0}(w)\right), \\
& \lim _{n \rightarrow \infty} g\left(w, \eta_{n}(w)\right)=g\left(w, \vartheta_{0}(w)\right), \\
& \lim _{n \rightarrow \infty} g\left(w, \zeta_{n}(w)\right)=g\left(w, \mu_{0}(w)\right), \\
& \lim _{n \rightarrow \infty} g\left(w, \rho_{n}(w)\right)=g\left(w, v_{0}(w)\right) .
\end{aligned}
$$

Since $g\left(w, \theta_{0}(w)\right), g\left(w, \vartheta_{0}(w)\right), g\left(w, \mu_{0}(w)\right)$, and $g\left(w, v_{0}(w)\right)$ are measurable, then the functions $\xi(w), \eta(w), \zeta(w)$, and $\rho(w)$, defined by

$$
\begin{aligned}
& \xi(w)=g\left(w, \theta_{0}(w)\right), \\
& \eta(w)=g\left(w, \vartheta_{0}(w)\right), \\
& \zeta(w)=g\left(w, \mu_{0}(w)\right), \\
& \rho(w)=g\left(w, v_{0}(w)\right),
\end{aligned}
$$

are measurable too. Thus

$$
\begin{array}{ll}
\lim _{n \rightarrow \infty} g\left(w, \xi_{n}(w)\right)=\xi(w), & \lim _{n \rightarrow \infty} g\left(w, \eta_{n}(w)\right)=\eta(w), \\
\lim _{n \rightarrow \infty} g\left(w, \zeta_{n}(w)\right)=\zeta(w), & \lim _{n \rightarrow \infty} g\left(w, \rho_{n}(w)\right)=\rho(w) .
\end{array}
$$

Since $g$ is continuous, (48) implies that

$$
\begin{aligned}
& \lim _{n \rightarrow \infty} g\left(w, g\left(w, \xi_{n}(w)\right)\right)=g(w, \xi(w)), \\
& \lim _{n \rightarrow \infty} g\left(w, g\left(w, \eta_{n}(w)\right)\right)=g(w, \eta(w)), \\
& \lim _{n \rightarrow \infty} g\left(w, g\left(w, \zeta_{n}(w)\right)\right)=g(w, \zeta(w)), \\
& \lim _{n \rightarrow \infty} g\left(w, g\left(w, \rho_{n}(w)\right)\right)=g(w, \rho(w)) .
\end{aligned}
$$

From (22) and commutativity of $F$ and $g$, we have

$$
\begin{aligned}
F( & \left(w,\left(w, \xi_{n}(w)\right), g\left(w, \eta_{n}(w)\right)\right. \\
& \left.\left.g\left(w, \zeta_{n}(w)\right), g\left(w, \rho_{n}(w)\right)\right)\right) \\
= & g\left(w, F\left(w,\left(\xi_{n}(w), \eta_{n}(w), \zeta_{n}(w), \rho_{n}(w)\right)\right)\right) \\
= & g\left(w, g\left(w, \xi_{n+1}(w)\right)\right)
\end{aligned}
$$

$$
\begin{aligned}
& F\left(w,\left(g\left(w, \eta_{n}(w)\right), g\left(w, \zeta_{n}(w)\right),\right.\right.\left.\left.g\left(w, \rho_{n}(w)\right), g\left(w, \xi_{n}(w)\right)\right)\right) \\
&= g\left(w, F\left(w,\left(\eta_{n}(w), \zeta_{n}(w), \rho_{n}(w), \xi_{n}(w)\right)\right)\right) \\
&= g\left(w, g\left(w, \eta_{n+1}(w)\right)\right), \\
& F\left(w,\left(g\left(w, \zeta_{n}(w)\right), g\left(w, \rho_{n}(w)\right),\right.\right. \\
&\left.\left.\quad g\left(w, \xi_{n}(w)\right), g\left(w, \eta_{n}(w)\right)\right)\right) \\
&=g\left(w, F\left(w,\left(\zeta_{n}(w), \rho_{n}(w), \xi_{n}(w), \eta_{n}(w)\right)\right)\right) \\
&=g\left(w, g\left(w, \zeta_{n+1}(w)\right)\right), \\
& F\left(w,\left(g\left(w, \rho_{n}(w)\right), g\left(w, \xi_{n}(w)\right),\right.\right. \\
&=\left.\left.g\left(w, \eta_{n}(w)\right), g\left(w, \zeta_{n}(w)\right)\right)\right) \\
&= g\left(w, F\left(w,\left(\rho_{n}(w), \xi_{n}(w), \eta_{n}(w), \zeta_{n}(w)\right)\right)\right) \\
&= g\left(w, g\left(w, \rho_{n+1}(w)\right)\right) .
\end{aligned}
$$

Now, we will show that if the assumption (a) or (b) holds, then

$$
\begin{aligned}
& F(w,(\xi(w), \eta(w), \zeta(w), \rho(w)))=g(w, \xi(w)) \\
& F(w,(\eta(w), \zeta(w), \rho(w), \xi(w)))=g(w, \eta(w)) \\
& F(w,(\zeta(w), \rho(w), \xi(w), \eta(w)))=g(w, \zeta(w)) \\
& F(w,(\rho(w), \xi(w), \eta(w), \zeta(w)))=g(w, \rho(w))
\end{aligned}
$$

for all $w \in \Omega$. Suppose (a) holds; then from (48), (49), (50), and the continuity of $F$, we obtain

$$
\begin{aligned}
& g(w, \xi(w)) \\
& =\lim _{n \rightarrow \infty} g\left(w, g\left(w, \xi_{n+1}(w)\right)\right) \\
& =\lim _{n \rightarrow \infty} F\left(w,\left(g\left(w, \xi_{n}(w)\right), g\left(w, \eta_{n}(w)\right),\right.\right. \\
& =F\left(w,\left(\lim _{n \rightarrow \infty} g\left(w, \xi_{n}(w)\right), \lim _{n \rightarrow \infty} g\left(w, \eta_{n}(w)\right),\right.\right. \\
& \left.\left.=\lim _{n \rightarrow \infty} g\left(w, \zeta_{n}(w)\right), \lim _{n \rightarrow \infty} g\left(w, \rho_{n}(w)\right)\right)\right) \\
& =F(w,(\xi(w), \eta(w), \zeta(w), \rho(w))),
\end{aligned}
$$


and similarly

$$
\begin{aligned}
& g(w, \eta(w)) \\
& =\lim _{n \rightarrow \infty} g\left(w, g\left(w, \eta_{n+1}(w)\right)\right) \\
& =\lim _{n \rightarrow \infty} F\left(w,\left(g\left(w, \eta_{n}(w)\right), g\left(w, \zeta_{n}(w)\right),\right.\right. \\
& \left.\left.g\left(w, \rho_{n}(w)\right), g\left(w, \xi_{n}(w)\right)\right)\right) \\
& =F\left(w,\left(\lim _{n \rightarrow \infty} g\left(w, \eta_{n}(w)\right), \lim _{n \rightarrow \infty} g\left(w, \zeta_{n}(w)\right),\right.\right. \\
& \left.\left.\lim _{n \rightarrow \infty} g\left(w, \rho_{n}(w)\right), \lim _{n \rightarrow \infty} g\left(w, \xi_{n}(w)\right)\right)\right) \\
& =F(w,(\eta(w), \zeta(w), \rho(w), \xi(w))), \\
& g(w, \zeta(w)) \\
& =\lim _{n \rightarrow \infty} g\left(w, g\left(w, \zeta_{n+1}(w)\right)\right) \\
& =\lim _{n \rightarrow \infty} F\left(w,\left(g\left(w, \zeta_{n}(w)\right), g\left(w, \rho_{n}(w)\right),\right.\right. \\
& \left.\left.g\left(w, \xi_{n}(w)\right), g\left(w, \eta_{n}(w)\right)\right)\right) \\
& =F\left(w,\left(\lim _{n \rightarrow \infty} g\left(w, \eta_{n}(w)\right), \lim _{n \rightarrow \infty} g\left(w, \zeta_{n}(w)\right),\right.\right. \\
& \left.\left.\lim _{n \rightarrow \infty} g\left(w, \rho_{n}(w)\right), \lim _{n \rightarrow \infty} g\left(w, \xi_{n}(w)\right)\right)\right) \\
& =F(w,(\eta(w), \zeta(w), \rho(w), \xi(w))), \\
& g(w, \rho(w)) \\
& =\lim _{n \rightarrow \infty} g\left(w, g\left(w, \rho_{n+1}(w)\right)\right) \\
& =\lim _{n \rightarrow \infty} F\left(w,\left(g\left(w, \rho_{n}(w)\right), g\left(w, \xi_{n}(w)\right),\right.\right. \\
& \left.\left.g\left(w, \eta_{n}(w)\right), g\left(w, \zeta_{n}(w)\right)\right)\right) \\
& =F\left(w,\left(\lim _{n \rightarrow \infty} g\left(w, \rho_{n}(w)\right), \lim _{n \rightarrow \infty} g\left(w, \xi_{n}(w)\right),\right.\right. \\
& \left.\left.\lim _{n \rightarrow \infty} g\left(w, \eta_{n}(w)\right), \lim _{n \rightarrow \infty} g\left(w, \zeta_{n}(w)\right)\right)\right) \\
& =F(w,(\rho(w), \xi(w), \eta(w), \zeta(w))) \text {. }
\end{aligned}
$$

Thus, we proved that $(\xi(w), \eta(w), \zeta(w), \rho(w)) \in X^{4}$ is a quadruple random coincidence of $F$ and $g$.

Suppose, now, the assumption (b) holds. Since $\left\{g\left(w, \xi_{n}(w)\right)\right\}$ and $\left\{g\left(w, \zeta_{n}(w)\right)\right\}$ are nondecreasing and $g\left(w, \xi_{n}(w)\right) \rightarrow g\left(w, \theta_{0}(w)\right)=\xi(w), g\left(w, \zeta_{n}(w)\right) \rightarrow$ $g\left(w, \mu_{0}(w)\right)=\zeta(w)$ and also $\left\{g\left(w, \eta_{n}(w)\right)\right\}$ and $\left\{g\left(w, \rho_{n}(w)\right)\right\}$ are nonincreasing and $g\left(w, \eta_{n}(w)\right) \rightarrow g\left(w, \vartheta_{0}(w)\right)=\eta(w)$, $g\left(w, \rho_{n}(w)\right) \rightarrow g\left(w, v_{0}(w)\right)=\rho(w)$, then by the assumption (b), we have

$$
\begin{aligned}
& g\left(w, \xi_{n}(w)\right) \leq g\left(w, \theta_{0}(w)\right)=\xi(w), \\
& g\left(w, \eta_{n}(w)\right) \geq g\left(w, \vartheta_{0}(w)\right)=\eta(w),
\end{aligned}
$$

$$
\begin{aligned}
& g\left(w, \zeta_{n}(w)\right) \leq g\left(w, \mu_{0}(w)\right)=\zeta(w), \\
& g\left(w, \rho_{n}(w)\right) \geq g\left(w, \nu_{0}(w)\right)=\rho(w) .
\end{aligned}
$$

Therefore, by the triangle inequality, (50), and (17), we have

$$
\begin{aligned}
& d(g(w, \xi(w)), F(w,(\xi(w), \eta(w), \zeta(w), \rho(w)))) \\
& \leq d\left(g(w, \xi(w)), g\left(w, g\left(w, \xi_{n+1}(w)\right)\right)\right) \\
&+ d\left(g\left(w, g\left(w, \xi_{n+1}(w)\right)\right)\right. \\
&F(w,(\xi(w), \eta(w), \zeta(w), \rho(w)))) \\
&= d\left(g(w, \xi(w)), g\left(w, g\left(w, \xi_{n+1}(w)\right)\right)\right) \\
&+ d\left(F \left(w,\left(g\left(w, \xi_{n}(w)\right), g\left(w, \eta_{n}(w)\right)\right)\right.\right. \\
&\left.\left.g\left(w, \zeta_{n}(w)\right), g\left(w, \rho_{n}(w)\right)\right)\right) \\
&F(w,(\xi(w), \eta(w), \zeta(w), \rho(w)))) \\
& \leq d\left(g(w, \xi(w)), g\left(w, g\left(w, \xi_{n+1}(w)\right)\right)\right) \\
&+\varphi\left(\frac { 1 } { 4 } \left[d\left(g\left(w, g\left(w, \xi_{n}(w)\right)\right), g(w, \xi(w))\right)\right.\right. \\
&+d\left(g\left(w, g\left(w, \eta_{n}(w)\right)\right), g(w, \eta(w))\right) \\
&+d\left(g\left(w, g\left(w, \zeta_{n}(w)\right)\right), g(w, \zeta(w))\right) \\
&\left.\left.+d\left(g\left(w, g\left(w, \rho_{n}(w)\right)\right), g(w, \rho(w))\right)\right]\right)
\end{aligned}
$$

and since $\varphi(t)<t$, we have

$$
\begin{aligned}
d(g(w, \xi(w)), F(w,(\xi(w), \eta(w), \zeta(w), \rho(w)))) \\
\leq d\left(g(w, \xi(w)), g\left(w, g\left(w, \xi_{n+1}(w)\right)\right)\right) \\
+\frac{1}{4}\left[d\left(g\left(w, g\left(w, \xi_{n}(w)\right)\right), g(w, \xi(w))\right)\right. \\
+d\left(g\left(w, g\left(w, \eta_{n}(w)\right)\right), g(w, \eta(w))\right) \\
+d\left(g\left(w, g\left(w, \zeta_{n}(w)\right)\right), g(w, \zeta(w))\right) \\
\left.+d\left(g\left(w, g\left(w, \rho_{n}(w)\right)\right), g(w, \rho(w))\right)\right] .
\end{aligned}
$$

Letting $n \rightarrow \infty$ and by (49), we get $d(g(w, \xi(w)), F(w,(\xi(w)$, $\eta(w), \zeta(w), \rho(w)))) \leq 0$. Hence

$$
F(w,(\xi(w), \eta(w), \zeta(w), \rho(w)))=g(w, \xi(w)) .
$$

Similarly, we can show that

$$
\begin{aligned}
& F(w,(\eta(w), \zeta(w), \rho(w), \xi(w)))=g(w, \eta(w)) \\
& F(w,(\zeta(w), \rho(w), \xi(w), \eta(w)))=g(w, \zeta(w)) \\
& F(w,(\rho(w), \xi(w), \eta(w), \zeta(w)))=g(w, \rho(w))
\end{aligned}
$$

for all $w \in \Omega$.

Thus we showed that $(\xi(w), \eta(w), \zeta(w), \rho(w)) \in X^{4}$ is a quadruple random coincidence of $F$ and $g$. 
As a consequence of Theorem 13, we can obtain the following theorem.

Theorem 14. Let $(X, d)$ be a complete separable metric space, and let $(\Omega, \Sigma)$ be a measurable space, and $F: \Omega \times X^{4} \rightarrow X$ has the mixed monotone property such that

(1) $F(w, \cdot)$ are continuous for all $w \in \Omega$,

(2) $F(\cdot, u)$ are measurable for all $u \in X^{4}$,

(3) there exists a $k \in[0,1)$ such that $F$ satisfies the following:

$$
\begin{aligned}
& d(F(w,(x, y, z, s)), F(w,(u, v, r, t))) \\
& \quad \leq k\left(\frac{d(x, u)+d(y, v)+d(z, r)+d(s, t)}{4}\right)
\end{aligned}
$$

for all $x, y, z, s, u, v, r, t \in X$ for which $x \leq u, y \geq v, z \leq r$, and $s \geq t$. Suppose either

(a) $F$ is continuous or

(b) X has the following property:

(1) if a nondecreasing sequence $\left\{x_{n}\right\} \rightarrow x$ then $x_{n} \leq$ $x$ for all $n$,

(2) if a nonincreasing sequence $\left\{y_{n}\right\} \rightarrow y$ then $y_{n} \geq$ $y$ for all $n$.

If there exist measurable mappings $\xi_{0}, \eta_{0}, \zeta_{0}, \rho_{0}: \Omega \rightarrow X$ such that

$$
\begin{array}{r}
\xi_{0}(w) \leq F\left(w,\left(\xi_{0}(w), \eta_{0}(w), \zeta_{0}(w), \rho_{0}(w)\right)\right), \\
\eta_{0}(w) \geq F\left(w,\left(\eta_{0}(w), \zeta_{0}(w), \rho_{0}(w), \xi_{0}(w)\right)\right), \\
\zeta_{0}(w) \leq F\left(w,\left(\zeta_{0}(w), \rho_{0}(w), \xi_{0}(w), \eta_{0}(w)\right)\right), \\
\rho_{0}(w) \geq F\left(w,\left(\rho_{0}(w), \xi_{0}(w), \eta_{0}(w), \zeta_{0}(w)\right)\right), \\
\text { for all } w \in \Omega,
\end{array}
$$

then there are measurable mappings $\xi, \eta, \zeta, \theta: \Omega \rightarrow X$ such that

$$
\begin{aligned}
& F(w,(\xi(w), \eta(w), \zeta(w), \rho(w)))=\xi(w), \\
& F(w,(\eta(w), \zeta(w), \rho(w), \xi(w)))=\eta(w), \\
& F(w,(\zeta(w), \rho(w), \xi(w), \eta(w)))=\zeta(w), \\
& F(w,(\rho(w), \xi(w), \eta(w), \zeta(w)))=\rho(w),
\end{aligned}
$$

for all $w \in \Omega$; that is, $F$ has a quadruple random fixed point.

Proof. Taking $\varphi(t)=k t$ with $k \in[0,1)$ and defining $g: \Omega \times$ $X \rightarrow X$ by $g(w, x)=x$ for all $w \in \Omega$ in Theorem 13, then we obtain Theorem 14.

In the following we prove the uniqueness of quadruple random fixed point.

We say that $(x, y, z, s)$ is equal to $(u, v, r, t)$ if and only if $x=u, y=v, z=r$, and $s=t$.
Theorem 15. In addition to the hypothesis of Theorem 13, assume that for all measurable mappings $x, y, z, s: \Omega \rightarrow X$ and $u, v, r, t: \Omega \rightarrow X$, there exist measurable mappings $a, b, c, d: \Omega \rightarrow X$ such that

$$
\begin{gathered}
(F(w,(a(w), b(w), c(w), d(w))), \\
F(w,(a(w), d(w), c(w), b(w))), \\
F(w,(c(w), b(w), a(w), d(w))), \\
F(w,(c(w), d(w), a(w), b(w))))
\end{gathered}
$$

is comparable to

$$
\begin{gathered}
(F(w,(x(w), y(w), z(w), s(w))), \\
F(w,(x(w), s(w), z(w), y(w))), \\
F(w,(z(w), y(w), x(w), s(w))), \\
F(w,(z(w), s(w), x(w), y(w)))), \\
(F(w,(u(w), v(w), r(w), t(w))), \\
F(w,(u(w), t(w), r(w), v(w))), \\
F(w,(t(w), v(w), u(w), r(w))), \\
F(w,(r(w), t(w), u(w), v(w))))
\end{gathered}
$$

Then $F$ and $g$ have a unique quadruple common random fixed point.

Proof. By Theorem 13, the set of quadruple common random fixed points of $F$ and $g$ is not empty. Suppose that $(x(w), y(w), z(w), s(w)),(u(w), v(w), r(w), t(w))$ are quadruple common random fixed points of $F$ and $g$; that is,

$$
\begin{aligned}
& F(w,(x(w), y(w), z(w), s(w)))=g(w, x(w)) \\
& F(w,(x(w), s(w), z(w), y(w)))=g(w, y(w)) \\
& F(w,(z(w), y(w), x(w), s(w)))=g(w, z(w)), \\
& F(w,(z(w), s(w), x(w), y(w)))=g(w, s(w)), \\
& F(w,(u(w), v(w), r(w), t(w)))=g(w, u(w)), \\
& F(w,(u(w), t(w), r(w), v(w)))=g(w, v(w)), \\
& F(w,(r(w), v(w), u(w), t(w)))=g(w, r(w)), \\
& F(w,(r(w), t(w), u(w), v(w)))=g(w, t(w)) .
\end{aligned}
$$

We prove that $(g(w, x(w)), g(w, y(w)), g(w, z(w)), g(w$, $s(w)))$ and $(g(w, u(w)), g(w, v(w)), g(w, r(w)), g(w, t(w)))$ are equal. By assumption, there exists $(a(w), b(w), c(w), d(w))$ such that

$$
\begin{gathered}
(F(w,(a(w), b(w), c(w), d(w))), \\
F(w,(a(w), d(w), c(w), b(w))), \\
F(w,(c(w), b(w), a(w), d(w))), \\
F(w,(c(w), d(w), a(w), b(w))))
\end{gathered}
$$


is comparable to

$$
\begin{gathered}
(F(w,(x(w), y(w), z(w), s(w))), \\
F(w,(x(w), s(w), z(w), y(w))), \\
F(w,(z(w), y(w), x(w), s(w))), \\
F(z(w), s(w), x(w), y(w))), \\
(F(w,(u(w), v(w), r(w), t(w))), \\
F(w,(u(w), t(w), r(w), v(w))), \\
F(w,(t(w), u(w), v(w), r(w))), \\
F(w,(r(w), t(w), u(w), v(w)))) .
\end{gathered}
$$

Define sequences $\left\{g\left(w, a_{n}(w)\right)\right\},\left\{g\left(w, b_{n}(w)\right)\right\},\left\{g\left(w, c_{n}(w)\right)\right\}$ and $\left\{g\left(w, d_{n}(w)\right)\right\}$ such that $a(w)=a_{0}(w), b(w)=b_{0}(w)$, $c(w)=c_{0}(w)$, and $d(w)=d_{0}(w)$ for all $n$, and

$$
\begin{aligned}
& g\left(w, a_{n}(w)\right) \\
& \quad=F\left(w,\left(a_{n-1}(w), b_{n-1}(w), c_{n-1}(w), d_{n-1}(w)\right)\right) \\
& g\left(w, b_{n}(w)\right) \\
& \quad=F\left(w,\left(a_{n-1}(w), d_{n-1}(w), c_{n-1}(w), b_{n-1}(w)\right)\right) \\
& g\left(w, c_{n}(w)\right) \\
& \quad=F\left(w,\left(c_{n-1}(w), b_{n-1}(w), a_{n-1}(w), d_{n-1}(w)\right)\right) \\
& g\left(w, d_{n}(w)\right) \\
& \quad=F\left(w,\left(c_{n-1}(w), d_{n-1}(w), a_{n-1}(w), b_{n-1}(w)\right)\right)
\end{aligned}
$$

Since (69) is comparable with (68), we can assume that $(g(w, x), g(w, y), g(w, z), g(w, s)) \quad \geq$ $(g(w, a(w)), g(w, b(w)), g(w, c(w)), g(w, d(w)))=$ $\left(g\left(w, a_{0}(w)\right), g\left(w, b_{0}(w)\right), g\left(w, c_{0}(w)\right), g\left(w, d_{0}(w)\right)\right)$; then, we get

$$
\begin{aligned}
& (g(w, x(w)), g(w, y(w)), g(w, z(w)), g(w, s(w))) \\
& \geq\left(g\left(w, a_{n}(w)\right), g\left(w, b_{n}(w)\right), g\left(w, c_{n}(w)\right), g\left(w, d_{n}(w)\right)\right)
\end{aligned}
$$

$$
\text { Using (72) and (17) }
$$

$$
\begin{aligned}
& d\left(g(w, x(w)), g\left(w, a_{n+1}(w)\right)\right) \\
& =d(F(w,(x(w), y(w), z(w), s(w))), \\
& \left.\quad F\left(w,\left(a_{n}(w), b_{n}(w), c_{n}(w), d_{n}(w)\right)\right)\right)
\end{aligned}
$$

$$
\begin{aligned}
& \leq \varphi\left(\left(d\left(g(w, x(w)), g\left(w, a_{n}(w)\right)\right)\right.\right. \\
& +d\left(g(w, y(w)), g\left(w, b_{n}(w)\right)\right) \\
& +d\left(g(w, z(w)), g\left(w, c_{n}(w)\right)\right) \\
& \left.\left.+d\left(g(w, s(w)), g\left(w, d_{n}(w)\right)\right)\right) \times(4)^{-1}\right), \\
& d\left(g\left(w, b_{n+1}(w)\right), g(w, y(w))\right) \\
& =d\left(F\left(w,\left(a_{n}(w), d_{n}(w), c_{n}(w), b_{n}(w)\right)\right),\right. \\
& F(w,(x(w), s(w), z(w), y(w)))), \\
& \leq \varphi\left(\left(d\left(g\left(w, a_{n}(w)\right), g(w, x(w))\right)\right.\right. \\
& +d\left(g\left(w, d_{n}(w)\right), g(w, s(w))\right) \\
& +d\left(g\left(w, c_{n}(w)\right), g(w, z(w))\right) \\
& \left.\left.+d\left(g\left(w, b_{n}(w)\right), g(w, y(w))\right)\right) \times(4)^{-1}\right), \\
& d\left(g(w, z(w)), g\left(w, c_{n+1}(w)\right)\right) \\
& =d(F(w,(z(w), y(w), x(w), s(w))), \\
& \left.F\left(w,\left(c_{n}(w), b_{n}(w), a_{n}(w), d_{n}(w)\right)\right)\right) \\
& \leq \varphi\left(\left(d\left(g(w, z(w)), g\left(w, c_{n}(w)\right)\right)\right.\right. \\
& +d\left(g(w, y(w)), g\left(w, b_{n}(w)\right)\right) \\
& +d\left(g(w, x(w)), g\left(w, a_{n}(w)\right)\right) \\
& \left.\left.+d\left(g(w, s(w)), g\left(w, d_{n}(w)\right)\right)\right) \times(4)^{-1}\right), \\
& d\left(g\left(w, d_{n+1}(w)\right), g(w, s(w))\right) \\
& =d\left(F\left(w,\left(c_{n}(w), d_{n}(w), a_{n}(w), b_{n}(w)\right)\right),\right. \\
& F(w,(z(w), s(w), x(w), y(w)))) \\
& \leq \varphi\left(\left(d\left(g\left(w, c_{n}\right), g(w, z(w))\right)\right.\right. \\
& +d\left(g\left(w, d_{n}(w)\right), g(w, s(w))\right) \\
& +d\left(g\left(w, a_{n}(w)\right), g(w, x(w))\right) \\
& \left.\left.+d\left(g\left(w, b_{n}(w)\right), g(w, y(w))\right)\right) \times(4)^{-1}\right) \text {. }
\end{aligned}
$$

Set

$$
\begin{aligned}
\delta_{n}= & d\left(g(w, x(w)), g\left(w, a_{n}(w)\right)\right) \\
& +d\left(g(w, y(w)), g\left(w, b_{n}(w)\right)\right) \\
& +d\left(g(w, z(w)), g\left(w, c_{n}(w)\right)\right) \\
& +d\left(g(w, s(w)), g\left(w, d_{n}(w)\right)\right) .
\end{aligned}
$$

From (73)

$$
\frac{\delta_{n+1}}{4} \leq \varphi\left(\frac{\delta_{n}}{4}\right) \quad \text { for all } n \geq 0
$$


which implies

$$
\frac{\delta_{n+1}}{4} \leq \varphi^{n}\left(\frac{\delta_{1}}{4}\right) \text { for all } n \geq 0 .
$$

Since $\varphi(t)<t$ and $\lim _{r \rightarrow t^{+}} \varphi(r)<t$ then $\lim _{n \rightarrow \infty} \varphi(t)=0$ for all $t>0$. Therefore (69) implies that

$$
\begin{aligned}
& \lim _{n \rightarrow \infty} d\left(g(w, x(w)), g\left(w, a_{n}(w)\right)\right)=0, \\
& \lim _{n \rightarrow \infty} d\left(g(w, y(w)), g\left(w, b_{n}(w)\right)\right)=0, \\
& \lim _{n \rightarrow \infty} d\left(g(w, z(w)), g\left(w, c_{n}(w)\right)\right)=0, \\
& \lim _{n \rightarrow \infty} d\left(g(w, s(w)), g\left(w, d_{n}(w)\right)\right)=0 .
\end{aligned}
$$

Similarly, we can show that

$$
\begin{aligned}
& \lim _{n \rightarrow \infty} d\left(g(w, u(w)), g\left(w, a_{n}(w)\right)\right)=0, \\
& \lim _{n \rightarrow \infty} d\left(g(w, v(w)), g\left(w, b_{n}(w)\right)\right)=0, \\
& \lim _{n \rightarrow \infty} d\left(g(w, r(w)), g\left(w, c_{n}(w)\right)\right)=0, \\
& \lim _{n \rightarrow \infty} d\left(g(w, t(w)), g\left(w, d_{n}(w)\right)\right)=0 .
\end{aligned}
$$

Combining (77) and (78) and using triangle inequality,

$$
\begin{aligned}
& d(g(w, u(w)), g(w, x(w))) \\
& \leq d\left(g(w, u(w)), g\left(w, a_{n}(w)\right)\right) \\
& \quad+d\left(g\left(w, a_{n}(w)\right), g(w, x(w))\right) \\
& \quad \longrightarrow 0 \text { as } n \longrightarrow \infty,
\end{aligned}
$$$$
d(g(w, v(w)), g(w, y(w)))
$$$$
\leq d\left(g(w, v(w)), g\left(w, b_{n}(w)\right)\right)
$$$$
+d\left(g\left(w, b_{n}(w)\right), g(w, y(w))\right)
$$$$
\longrightarrow 0 \text { as } n \longrightarrow \infty \text {, }
$$$$
d(g(w, r(w)), g(w, z(w)))
$$$$
\leq d\left(g(w, r(w)), g\left(w, c_{n}(w)\right)\right)
$$$$
+d\left(g\left(w, c_{n}(w)\right), g(w, z(w))\right)
$$$$
\longrightarrow 0 \text { as } n \longrightarrow \infty \text {, }
$$$$
d(g(w, t(w)), g(w, s(w)))
$$$$
\leq d\left(g(w, t(w)), g\left(w, d_{n}(w)\right)\right)
$$$$
+d\left(g\left(w, d_{n}(w)\right), g(w, t(w))\right)
$$$$
\longrightarrow 0 \quad \text { as } n \longrightarrow \infty \text {. }
$$

Therefore

$$
\begin{aligned}
& (g(w, x(w)), g(w, y(w)), g(w, z(w)), g(w, s(w))) \\
& \quad=(g(w, u(w)), g(w, v(w)), g(w, r(w)), g(w, t(w))) .
\end{aligned}
$$

By commutativity of $F$ and $g$ and using (67), we get

$$
\begin{aligned}
g(w, g(w, x(w))) & \\
= & g(w, F(w,(x(w), y(w), z(w), s(w)))) \\
= & F(w,(g(w, x(w)), g(w, y(w)), \\
& \quad g(w, z(w)), g(w, s(w)))), \\
g(w, g(w, y(w))) & g(w, F(w,(x(w), s(w), z(w), y(w)))) \\
= & F(w,(g(w, x(w)), g(w, s(w)), \\
& \quad g(w, z(w)), g(w, y(w)))), \\
g(w, g(w, z(w))) & g(w, F(w,(z(w), y(w), x(w), s(w)))) \\
= & F(w,(g(w, z(w)), g(w, y(w)), \\
& \quad g(w, x(w)), g(w, s(w)))), \\
g(w, g(w, s(w))) & g(w, F(w,(z(w), s(w), x(w), y(w)))) \\
= & F(w,(g(w, z(w)), g(w, s(w)),
\end{aligned}
$$

Set

$$
\begin{array}{ll}
g(w, x(w))=\xi(w), & g(w, y(w))=\eta(w), \\
g(w, z(w))=\zeta(w), & g(w, s(w))=\rho(w) .
\end{array}
$$

Then, (81) becomes

$$
\begin{aligned}
& g(w, \xi(w))=F(w,(\xi(w), \eta(w), \zeta(w), \rho(w))), \\
& g(w, \eta(w))=F(w,(\xi(w), \rho(w), \zeta(w), \eta(w))), \\
& g(w, \zeta(w))=F(w,(\zeta(w), \eta(w), \xi(w), \rho(w))), \\
& g(w, \rho(w))=F(w,(\zeta(w), \rho(w), \xi(w), \eta(w))) .
\end{aligned}
$$

Thus, $(\xi(w), \eta(w), \zeta(w), \rho(w))$ is a quadruple common random coincidence point of $F$ and $g$. Putting $(u(w), v(w), r(w), t(w))=(\xi(w), \eta(w), \zeta(w), \rho(w))$ in (80), we have

$g(w, x(w))=g(w, \xi(w)), \quad g(w, y(w))=g(w, \eta(w))$,

$g(w, z(w))=g(w, \zeta(w)), \quad g(w, s(w))=g(w, \rho(w))$. 
From (82), (84), and (83), we have

$$
\begin{aligned}
& \xi(w)=g(w, \xi(w))=F(w,(\xi(w), \eta(w), \zeta(w), \rho(w))) \\
& \eta(w)=g(w, \eta(w))=F(w,(\xi(w), \rho(w), \zeta(w), \eta(w))) \\
& \zeta(w)=g(w, \zeta(w))=F(w,(\zeta(w), \eta(w), \xi(w), \rho(w))) \\
& \rho(w)=g(w, \rho(w))=F(w,(\zeta(w), \rho(w), \xi(w), \eta(w)))
\end{aligned}
$$

Thus, ( $\xi(w), \eta(w), \zeta(w), \rho(w))$ is a quadruple common random fixed point of $F$ and $g$. Due to (80), it is unique.

\section{Examples}

In this section we give some examples to show that our results are effective.

Example 16. Let $X=\mathbb{R}$ with the usual ordering and usual metric. Let $\Omega=[0,1]$ and let $\Sigma$ be the sigma algebra of Lebesgue's measurable subset of $[0,1]$.

Define $g: \Omega \times X \rightarrow X, F: \Omega \times X^{4} \rightarrow X$ and $\varphi:$ $[0, \infty) \rightarrow[0, \infty)$ as follows.

$g(w, x)=\left(3\left(1-w^{2}\right) / 4\right) x, F(w,(x, y, z, s))=((1-$ $\left.\left.w^{2}\right) / 8\right)(x-y+z-s), w \in \Omega$, and $\phi(t)=(2 / 3) t$ for all $t \in[0, \infty)$. We will check that the contraction (17) is satisfied for all $x, y, z, s, u, v, r, t \in X$ satisfying $g(w, x) \leq g(w, u)$, $g(w, y) \geq g(w, v), g(w, z) \leq g(w, r)$, and $g(w, s) \geq g(w, t)$ for all $w \in \Omega$.

The left-hand side (L.H.S.) of (17) is

$$
\begin{gathered}
d(F(w,(x, y, z, s)), F(w,(u, v, r, t))) \\
=d\left(\frac{\left(1-w^{2}\right)}{8}(x-y+z-s),\right. \\
\left.\frac{\left(1-w^{2}\right)}{8}(u-v+r-t)\right) \\
=\frac{\left(1-w^{2}\right)}{8} \mid(x-u)+(v-y) \\
+(z-r)+(t-s) \mid \\
\leq \frac{\left(1-w^{2}\right)}{8}(|(x-u)|+|(y-v)| \\
+|(z-r)|+|(s-t)|) .
\end{gathered}
$$

The right-hand side (R.H.S.) of (17) is

$$
\begin{aligned}
& \varphi((d(g(w, x), g(w, u))+d(g(w, y), g(w, v)) \\
& \left.\quad+d(g(w, z), g(w, r))+d(g(w, s), g(w, t)) \times(4)^{-1}\right)
\end{aligned}
$$

$$
\begin{aligned}
& =\frac{2}{3}((d(g(w, x), g(w, u))+d(g(w, y), g(w, v)) \\
& +d(g(w, z), g(w, r)) \\
& \left.+d(g(w, s), g(w, t))) \times(4)^{-1}\right) \\
& =\frac{2}{3} \times \frac{1}{4} \times \frac{3\left(1-w^{2}\right)}{4}(|(x-u)|+|(y-v)| \\
& +|(z-r)|+|(s-t)|) \\
& =\frac{\left(1-w^{2}\right)}{8}(|(x-u)|+|(y-v)| \\
& +|(z-r)|+|(s-t)|) .
\end{aligned}
$$

Then L.H.S $\leq$ R.H.S.; that is, contraction (17) is satisfied. It is obvious that the other hypotheses of Theorem 13 are satisfied. We deduce that $(0,0,0,0)$ is the unique quadruple common random fixed point of $F$ and $g$.

Example 17. Let $X=\mathbb{R}$ with the usual ordering and usual metric. Let $\Omega=[0,1]$ and let $\Sigma$ be the sigma algebra of Lebesgue's measurable subset of $[0,1]$.

Define $g: \Omega \times X \rightarrow X, F: \Omega \times X^{4} \rightarrow X$ and $\varphi:$ $[0, \infty) \rightarrow[0, \infty)$ as follows:

$$
\begin{gathered}
g(w, x)=\frac{n+1}{n}\left(1-w^{2}\right) x, \quad n=1,2,3, \ldots, \\
F(w,(x, y, z, s))=\frac{\left(1-w^{2}\right)}{4} x, \\
\varphi(t)=\frac{n}{n+1} t .
\end{gathered}
$$

L.H.S

$$
\begin{aligned}
d & (F(w,(x, y, z, s)), F(w,(u, v, r, t))) \\
& =d\left(\left(1-w^{2}\right) x,\left(1-w^{2}\right) u\right) \\
& =\frac{\left(1-w^{2}\right)}{4}|(x-u)|
\end{aligned}
$$

R.H.S.

$$
\begin{aligned}
\varphi((d(g(w, x), g(w, u))+d(g(w, y), g(w, v)) \\
\left.+d(g(w, z), g(w, r))+d(g(w, s), g(w, t))) \times(4)^{-1}\right) \\
=\frac{n}{n+1}((d(g(w, x), g(w, u))+d(g(w, y), g(w, v)) \\
+d(g(w, z), g(w, r)) \\
\left.+d(g(w, s), g(w, t))) \times(4)^{-1}\right)
\end{aligned}
$$




$$
\begin{aligned}
= & \frac{n}{n+1} \times \frac{1}{4} \times \frac{n+1}{n}\left(1-w^{2}\right) \\
& \times(|(x-u)|+|(y-v)|+|(z-r)|+|(s-t)|) \\
= & \frac{\left(1-w^{2}\right)}{4}(|(x-u)|+|(y-v)|+|(z-r)|+|(s-t)|) .
\end{aligned}
$$

Thus it is verified that the functions $g, F$, and $\varphi$ satisfy all the conditions of Theorem 13 , and $(0,0,0,0)$ is the quadruple random coincidence of $g$ and $F$ in $X$.

\section{Conflict of Interests}

The authors declare that there is no conflict of interests regarding the publication of this paper.

\section{References}

[1] I. Beg and N. Shahzad, "Random fixed point theorems for nonexpansive and contractive-type random operators on Banach spaces," Journal of Applied Mathematics and Stochastic Analysis, vol. 7, no. 4, pp. 569-580, 1994.

[2] A. T. Bharucha-Reid, "Fixed point theorems in probabilistic analysis," Bulletin of the American Mathematical Society, vol. 82, no. 5, pp. 641-657, 1976.

[3] O. Hans, "Reduzierende zulliallige transformaten," Czechoslovak Mathematical Journal, vol. 7, no. 82, pp. 154-158, 1957.

[4] O. Hans, "Random operator equations," in Proceedings of the 4th Berkeley Symposium on Mathematical Statistics and Probability, vol. 2, pp. 185-202, University California Press, Berkeley, Calif, USA, 1961.

[5] C. J. Himmelberg, "Measurable relations," Fundamenta Mathematicae, vol. 87, pp. 53-72, 1975.

[6] S. Itoh, "A random fixed point theorem for a multivalued contraction mapping," Pacific Journal of Mathematics, vol. 68, no. 1, pp. 85-90, 1977.

[7] S. Itoh, "Random fixed-point theorems with an application to random differential equations in Banach spaces," Journal of Mathematical Analysis and Applications, vol. 67, no. 2, pp. 261273, 1979.

[8] N. S. Papageorgiou, "Random fixed point theorems for measurable multifunctions in Banach spaces," Proceedings of the American Mathematical Society, vol. 97, no. 3, pp. 507-514, 1986.

[9] R. T. Rockafellar, "Measurable dependence of convex sets and functions on parameters," Journal of Mathematical Analysis and Applications, vol. 28, pp. 4-25, 1969.

[10] A. Špaček, “Zufällige gleichungen," Czechoslovak Mathematical Journal, vol. 5, no. 80, pp. 462-466, 1955.

[11] D. H. Wagner, "Survey of measurable selection theorems," SIAM Journal on Control and Optimization, vol. 15, no. 5, pp. 859-903, 1977.

[12] H. K. Xu, "Some random fixed point theorems for condensing and nonexpansive operators," Proceedings of the American Mathematical Society, vol. 110, no. 2, pp. 395-400, 1990.

[13] M. Turinici, "Abstract comparison principles and multivariable Gronwall-Bellman inequalities," Journal of Mathematical Analysis and Applications, vol. 117, no. 1, pp. 100-127, 1986.

[14] A. C. M. Ran and M. C. B. Reurings, "A fixed point theorem in partially ordered sets and some applications to matrix equations," Proceedings of the American Mathematical Society, vol. 132, no. 5, pp. 1435-1443, 2004.

[15] T. G. Bhaskar and V. Lakshmikantham, "Fixed point theorems in partially ordered metric spaces and applications," Nonlinear Analysis: Theory, Methods \& Applications, vol. 65, no. 7, pp. 13791393, 2006.

[16] V. Lakshmikantham and L. B. Ćirić, "Coupled fixed point theorems for nonlinear contractions in partially ordered metric spaces," Nonlinear Analysis: Theory, Methods \& Applications, vol. 70, no. 12, pp. 4341-4349, 2009.

[17] L. B. Ćirić and V. Lakshmikantham, "Coupled random fixed point theorems for nonlinear contractions in partially ordered metric spaces," Stochastic Analysis and Applications, vol. 27, no. 6, pp. 1246-1259, 2009.

[18] V. Berinde and M. Borcut, "Tripled fixed point theorems for contractive type mappings in partially ordered metric spaces," Nonlinear Analysis: Theory, Methods \& Applications, vol. 74, no. 15, pp. 4889-4897, 2011.

[19] E. Karapinar, "Quartet fixed point for nonlinear contraction," submitted toGeneral Topology, 2011, http://arxiv.org/pdf/1106 .5472.pdf.

[20] E. Karapinar and V. Berinde, "Quadruple fixed point theorems for nonlinear contractions in partially ordered metric spaces," Banach Journal of Mathematical Analysis, vol. 6, no. 1, pp. 7489, 2012.

[21] E. Karapinar and N. V. Luong, "Quadruple fixed point theorems for nonlinear contractions," Computers \& Mathematics with Applications, vol. 64, no. 6, pp. 1839-1848, 2012.

[22] Z. Mustafa, H. Aydi, and E. Karapınar, "Mixed $g$-monotone property and quadruple fixed point theorems in partially ordered metric spaces," Fixed Point Theory and Applications, vol. 2012, article 71, 2012.

[23] E. Karapınar, W. Shatanawi, and Z. Mustafa, "Quadruple fixed point theorems under nonlinear contractive conditions in partially ordered metric spaces," Journal of Applied Mathematics, vol. 2012, Article ID 951912, 17 pages, 2012.

[24] E. J. McShane and R. B. Warfield, Jr., "On Filippov's implicit functions lemma," Proceedings of the American Mathematical Society, vol. 18, pp. 41-47, 1967. 


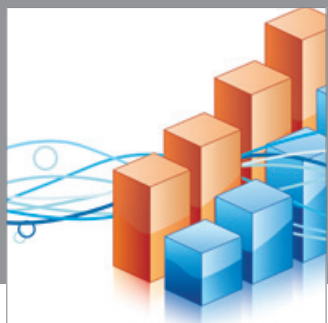

Advances in

Operations Research

mansans

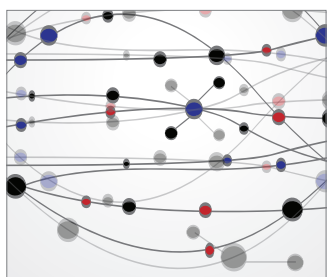

The Scientific World Journal
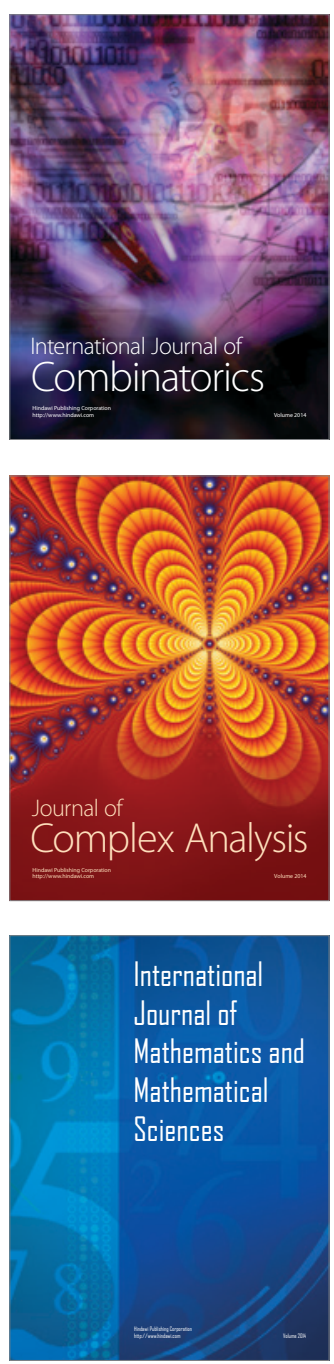
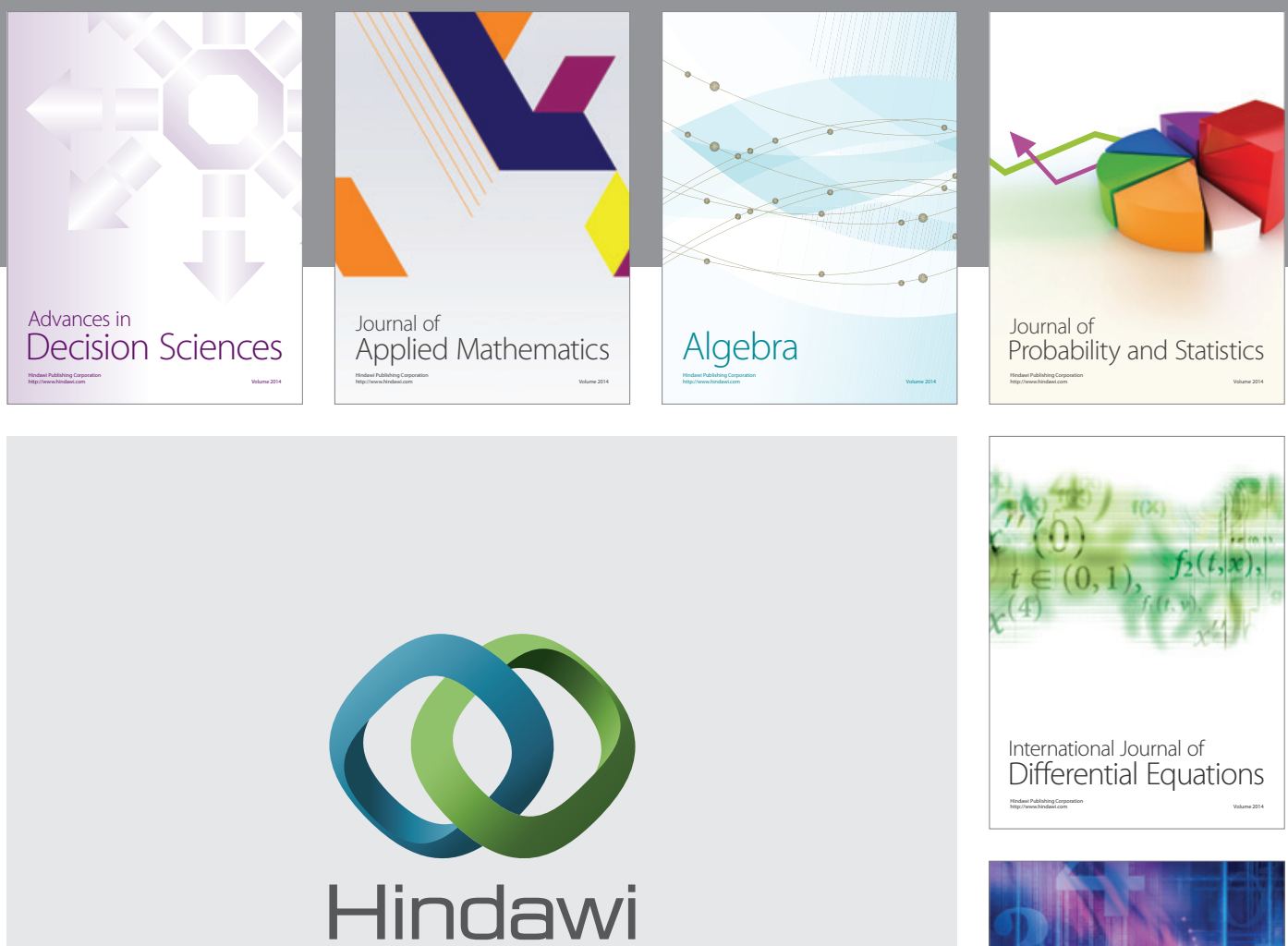

Submit your manuscripts at http://www.hindawi.com
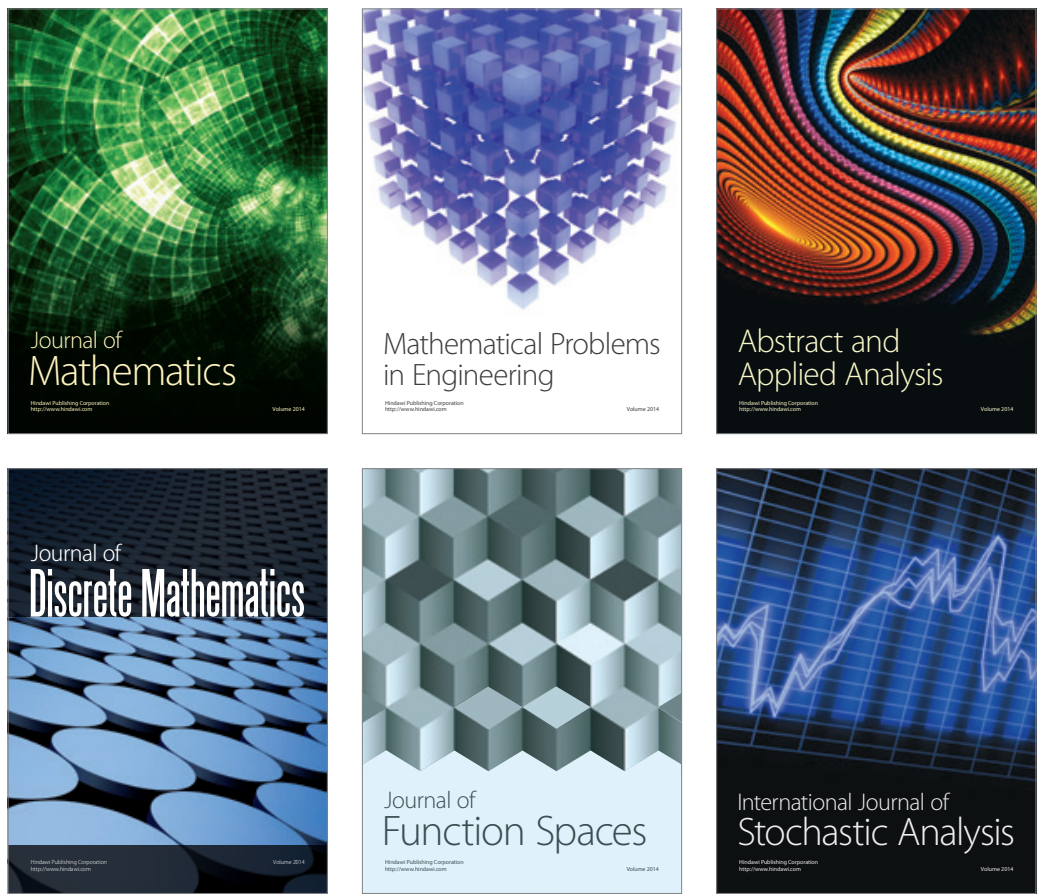

Journal of

Function Spaces

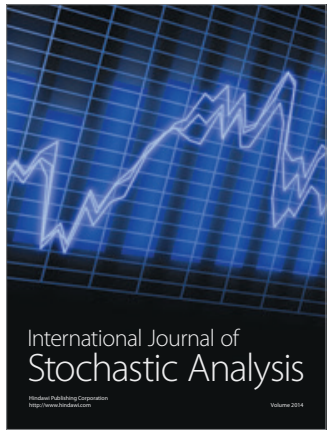

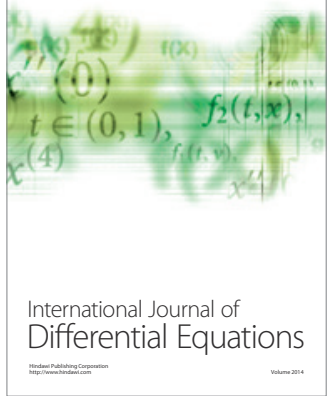
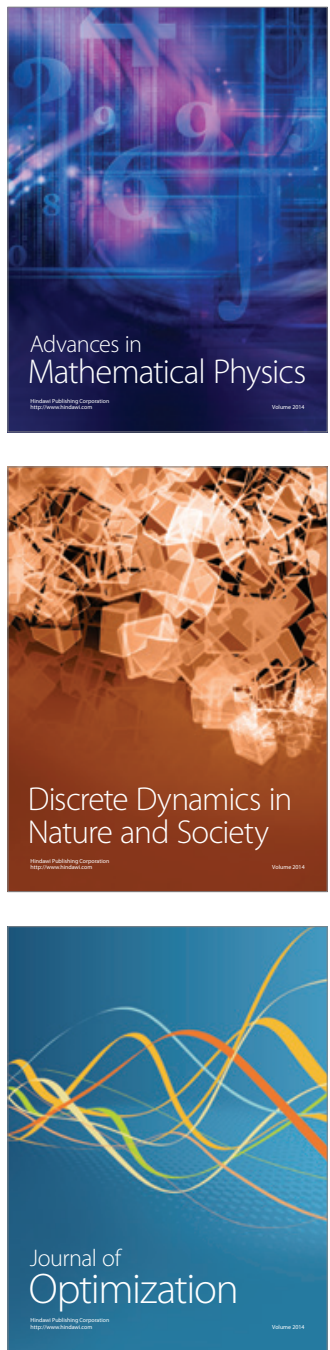